\title{
Almost sure exponential stability of dynamical systems driven by Lévy processes and its application to control design for magnetic bearings
}

\author{
K. D. Do \\ Department of Mechanical Engineering, Curtin University, Bentley, WA 6102, Australia \\ H. L. Nguyen \\ Department of Planning and Finance, Thai Nguyen University, Thai Nguyen City, Vietnam
}

\begin{abstract}
A Lyapunov-type theorem is developed to study well-posedness and almost surely $\mathcal{K}_{\infty}$-exponential stability of dynamical systems driven by Lévy processes. Sufficient conditions imposed by the theorem, which are relatively easy to be verified, make it applicable to control design and stability analysis. The theorem is then applied to design tracking controllers to achieve almost surely $\mathcal{K}$-exponential stability for magnetic bearings under diffuse-jump loads subject to an output tracking constraint.
\end{abstract}

Keywords: Lyapunov-type theorem; Well-posedness; Exponential Stability; Lévy processes; Magnetic bearings.

\section{Introduction}

Well-posedness (existence and uniqueness of the solution) and stability of stochastic differential equations (SDEs), which model dynamical systems under stochastic loads, have been extensively studied (e.g., (H. Deng, Krstic, \& Williams, 2001; Do, 2015, 2016; Khasminskii, 1980; Liu, 2006; X. Mao, 2007) on SDEs driven by diffuses (Brownian motions), (Applebaum, 2009; Applebaum \& Siakalli, 2009; W. Mao, You, \& Mao, 2016; X. Mao, 1999; X. Mao, Yin, \& Yuan, 2007; Teel, Subbaramana, \& Sferlazza, 2014; Zhu, 2014) on SDEs driven by diffuses and jumps, (Antunes, Hespanha, \& Silvestre, 2010, 2012, 2013a, 2013b, 2014; F. Deng, Luo, \& Mao, 2012; Hespanha \& Teel, 2006; Teel et al., 2014) on impulsive SDEs driven by renewal processes, which can be viewed as SDEs driven by diffuses and jumps via the Lévy-Itô decomposition (Applebaum, 2009, Theorem 2.4.16)). To study local/global well-posedness of SDEs, different Lipschitz and growth conditions are imposed on the system functions. Because one type of stability does not usually imply the other(s) in a stochastic setting (X. Mao, 2007), different criteria are proposed to guarantee different types of stability. Stability in moment and almost sure (a.s.) stability (sometime referred to as stability in probability) are often considered. Motivated by the fact that Lyapunov's direct method has been extensively used for deterministic dynamical system (Khalil, 2002), this method is also the most powerful tool to investigate stability in moment and a.s. stability of SDEs.

There are excessive and excellent results on Lyapunov sufficient conditions to ensure exponential stability in moment and a.s asymptotic stability of both SDEs driven by diffuses and jumps (Applebaum, 2009; Applebaum \& Siakalli, 2009; W. Mao et al., 2016; X. Mao, 1999; X. Mao et al., 2007; Teel et al., 2014; Zhu, 2014) and impulsive SDEs driven by renewal processes (Antunes et al., 2010, 2012, 2013a, 2013b, 2014; F. Deng et al., 2012; Hespanha \& Teel, 2006; Nane \& Ni, 2017; 
Teel et al., 2014). These conditions are analogous to those developed for deterministic differential equations, see (Khalil, 2002, Chapter 4), where an infinite generator should be used instead of the normal time derivative of a Lyapunov function. On the other hand, there are several results on a.s. exponential stability of SDEs driven by diffuses and jumps. In (Applebaum, 2009; Applebaum \& Siakalli, 2009), an important exponential martingale inequality is derived and the result on global a.s. exponential stability of SDES driven by diffuses in (X. Mao, 2007, Theorem 3.3) is generalized to SDEs driven by diffuses and jumps, where a negative logarithm condition on the jump term in the infinite generator of a Lyapunov function plus usual Lyapunov sufficient conditions are imposed, see (Applebaum \& Siakalli, 2009, Theorem 3.1), whose proof is the most interesting due to the use of the exponential martingale inequality that they derived. However, the negative logarithm condition on the jump term in the infinite generator creates difficulty in applying (Applebaum \& Siakalli, 2009, Theorem 3.1) to control design because the control is not able to change the above jump term in the infinite generator. In (W. Mao et al., 2016), the result on a.s. exponential stability of SDEs driven by diffuses and jumps is derived under a growth condition on the jump term, see (W. Mao et al., 2016, Theorem 3.2). This condition also creates difficulty in its application to control design. In (X. Mao et al., 2007), a linear growth condition is imposed on the jump term to prove a.s. exponential stability for SDEs with Markovian switching (or hybrid SDEs), see (X. Mao et al., 2007, Theorem 3.3). In (F. Deng et al., 2012), a different growth condition is imposed on the product of the Lyapunov function's gradient and the jump function to prove a.s. exponential stability for SDEs with Markovian switching, see (F. Deng et al., 2012, Theorem 2.2). In (X. Mao, 1999), a linear growth condition is imposed on the system function to prove that the exponential stability in moment implies the almost sure exponential stability for SDEs with Markovian switching, see (X. Mao, 1999, Theorem 3.2). The common feature in the aforementioned works for the study of a.s. exponential stability is the assumption made on the system jump function either independently or as a separate condition on the jump term of the Lyapunov function's infinite generator. From a control design point of view, it is convenient to derive sufficient conditions impose on a Lyapunov function and its (whole) infinite generator. Conditions imposed on the whole infinite generator are more relaxed than those on the individual jump term in the infinite generator in control design because the control can change the infinite generator on the whole but not its individual jump term. This is analogous to the results developed for deterministic systems, and has obtained for the study of exponential stability in moment and a.s. asymptotic stability for SDEs as reviewed above.

The above discussion motivates the first contribution of this paper on developing a Lyapunovtype theorem to study well-posedness and a.s. $\mathcal{K}_{\infty}$-exponential stability of systems driven by Lévy processes. We consider Lévy processes as disturbances driving the system because a Lévy process is preferably used in systems with high uncertainties, and can be decomposed to three components by Lévy-Itô decomposition (Applebaum, 2009): a drift, a Brownian motion, and a pure jump process, which can be used to correspondingly describe the deterministic, diffuse, and jump components. The Lyapunov conditions imposed by the theorem are similar to those required for exponential stability in moment and a.s. asymptotic stability for SDEs in the literature.

Based on well-posedness and stability results as discussed above, control of systems driven by diffuses and/or jumps has also been considered. When using the powerful Lyapunov direct method, control of systems driven by diffuses and jumps (in comparison with deterministic systems) has two difficulties induced by the Hessian and jump terms in the infinite generator of a Lyapunov function. Despite the difficulty caused by the Hessian term, control of systems driven by diffuses was considered by many authors (e.g., (H. Deng \& Krstic, 1997; H. Deng et al., 2001; Do, 2015)). However, the jump term is more complicated to be dealt with than the Hessian term. Thus, control of systems driven by both diffuses and jumps (Lévy processes) is a new area. The only reference is (Jagtap \& Zamani, 2017) on backstepping control design for Hamiltonian systems driven by diffuse-jumps to achieve stability in moment. As an application of the developed Lyapunov-type theorem for study of well-posedness and stability of systems driven by diffuses and jumps to control 
design, tracking controllers are designed for active magnetic bearings (AMBs) under stochastic (deterministic, diffusive, and jumped) loads. Control of AMBs under stochastic loads has not been addressed in the literature despite the fact that loads on the rotor of AMBs contain high uncertainties in a number of applications. Up to present, loads on the rotor of AMBs are only considered as (time-invariant or time-varying) deterministic disturbances. Related works include (Fujita, Hatake, \& Matsumura, 1993; Matsumura \& Yoshimoto, 1986; Mohamed \& Emad, 1992; Smith \& Weldon, 1995) on linear control, and (Charara, Miras, \& Caron, 1996; de Queiroz \& Dawson, 1996; de Queiroz, Dawson, \& Suri, 1998; Do, 2010; Mittal \& Menq, 1997; Smith \& Weldon, 1995; Torries, Sira-Ramirez, \& Escobar, 1999) on nonlinear control.

Motivated by the above review/discussion, the second contribution of this paper is the design of tracking controllers for AMBs driven by Lévy processes to achieve a.s. (practical) $\mathcal{K}$-exponential stability of the closed-loop system. The control design also imposes a hard constraint on the displacement tracking error.

Notations. The symbols $\wedge$ and $\vee$ denote the infimum and supremum operators, respectively. The symbol $\mathbb{E}$ denotes the expected value while $\mathbb{P}$ denotes probability.

\section{Well-posedness and Stability of SDEs}

This section presents SDEs to be considered, definition of a.s. stability, and a Lyapunov-type theorem on well-posedness and stability of SDEs. These results are to be used for control design and analysis of well-posedness and stability of magnetic bearings.

\section{$2.1 \quad$ Nonlinear SDEs}

Let $\boldsymbol{W}(t), t \geq t_{0} \geq 0$ be an $m_{1}$-dimensional standard Brownian motion vector defined on a complete probability space $(\Omega, \mathcal{F}, \mathbb{P})$ equipped with a filtration $\mathcal{F}_{t \geq t_{0}}$ such that it satisfies the usual hypotheses. Let $\boldsymbol{N}=\operatorname{col}\left(N_{1}, \ldots, N_{\ell}, \ldots, N_{m_{2}}\right)$ be an $m_{2}$-dimensional Poisson random measure vector defined on $\mathbb{R}^{+} \times\left(\mathbb{R}^{m_{2}}-\{0\}\right)$ with an $m_{2}$-dimensional compensator vector $\widetilde{\boldsymbol{N}}$ and $m_{2}$-dimensional intensity measure vector $\boldsymbol{\nu}=\operatorname{col}\left(\nu_{1}, \ldots, \nu_{\ell}, \ldots, \nu_{m_{2}}\right)$. We assume that all $N_{\ell}$ are independent of each other, $\boldsymbol{N}$ is independent of $\boldsymbol{W}$, and $\boldsymbol{\nu}$ is a Lévy measure vector such that $\widetilde{\boldsymbol{N}}(d t, d \boldsymbol{\xi}):=\boldsymbol{N}(d t, d \boldsymbol{\xi})-d t \boldsymbol{\nu}(d \boldsymbol{\xi})$ and $\int_{\mathbb{R}^{m_{2}-\{0\}}}\left(\|\boldsymbol{\xi}\|^{2} \wedge 1\right) \boldsymbol{\nu}(d \boldsymbol{\xi})<\infty$ with $\boldsymbol{\xi}=\operatorname{col}\left(\xi_{1}, \cdots, \xi_{\ell}, \cdots, \xi_{m_{2}}\right)$.

Consider the nonlinear SDE driven by Lévy processes:

$$
\begin{aligned}
& d \boldsymbol{X}(t)=\boldsymbol{F}(\boldsymbol{X}(t), t) d t+\boldsymbol{G}(\boldsymbol{X}(t), t) d \boldsymbol{W}(t)+\int_{\|\boldsymbol{\xi}\|<\varepsilon} \boldsymbol{\Phi}\left(\boldsymbol{X}\left(t^{-}\right), t, \boldsymbol{\xi}\right) \widetilde{\boldsymbol{N}}(d t, d \boldsymbol{\xi}), \\
& \boldsymbol{X}\left(t_{0}\right)=\boldsymbol{X}_{0} \in \mathbb{R}^{n},
\end{aligned}
$$

where $\boldsymbol{X}\left(t^{-}\right)=\lim _{s \uparrow t} \boldsymbol{X}(s) ; \boldsymbol{F}: \mathbb{R}^{n} \times\left[t_{0}, \infty\right) \rightarrow \mathbb{R}^{n} ; \boldsymbol{G}: \mathbb{R}^{n} \times\left[t_{0}, \infty\right) \rightarrow \mathbb{R}^{n \times m_{1}} ; \boldsymbol{\Phi}: \mathbb{R}^{n} \times\left[t_{0}, \infty\right) \times$ $\mathbb{R}^{m_{2}} \rightarrow \mathbb{R}^{n \times m_{2}}$, and $\int_{\|\boldsymbol{\xi}\|<\varepsilon} \boldsymbol{\Phi}\left(\boldsymbol{X}\left(t^{-}\right), t, \boldsymbol{\xi}\right) \widetilde{\boldsymbol{N}}(d t, d \boldsymbol{\xi}):=\sum_{\ell=1}^{m_{2}} \int_{\left|\xi_{\ell}\right|<\varepsilon_{\ell}} \boldsymbol{\Phi}^{(\ell)}\left(\boldsymbol{X}\left(t^{-}\right), t, \boldsymbol{\xi}\right) \widetilde{N}_{\ell}\left(d t, d \xi_{\ell}\right)$ with $\boldsymbol{\Phi}^{(\ell)}$ being the $\ell^{t h}$ column of $\boldsymbol{\Phi}$ for compact notation. The constants $\varepsilon_{\ell} \in(0, \infty]$ denote the maximum allowable jump sizes.

We assume that the system functions $\boldsymbol{F}(\boldsymbol{X}(t), t), \boldsymbol{G}(\boldsymbol{X}(t), t)$, and $\boldsymbol{\Phi}\left(\boldsymbol{X}\left(t^{-}\right), t, \boldsymbol{\xi}\right)$ satisfy the following local conditions to ensure local well-posedness of (1).

\section{Assumption 2.1:}

C1. [Local Lipschitz] For any $\boldsymbol{X}, \boldsymbol{Y} \in \mathbb{R}^{n}$ with $\|\boldsymbol{X}\| \vee\|\boldsymbol{Y}\| \leq \epsilon_{1}$, where $\epsilon_{1}$ is a positive constant, there exists a nonnegative constant $L_{1}$ such that for all $t \in\left[t_{0}, \infty\right)$ :

$$
\begin{aligned}
\|\boldsymbol{F}(\boldsymbol{X}, t)-\boldsymbol{F}(\boldsymbol{Y}, t)\|^{2} & +\|\boldsymbol{G}(\boldsymbol{X}, t)-\boldsymbol{G}(\boldsymbol{Y}, t)\|^{2} \\
& +\sum_{\ell=1}^{m_{2}} \int_{\left|\xi_{\ell}\right|<\varepsilon_{\ell}}\left\|\boldsymbol{\Phi}^{(\ell)}\left(\boldsymbol{X}, t, \xi_{\ell}\right)-\boldsymbol{\Phi}^{(\ell)}\left(\boldsymbol{Y}, t, \xi_{\ell}\right)\right\|^{2} \nu_{\ell}\left(d \xi_{\ell}\right) \leq L_{1}\|\boldsymbol{X}-\boldsymbol{Y}\|^{2} .
\end{aligned}
$$


C2. [Locally linear growth] For any $\boldsymbol{X} \in \mathbb{R}^{n}$ with $\|\boldsymbol{X}\| \leq \epsilon_{2}$, where $\epsilon_{2}$ is a positive constant, there exists a nonnegative constant $L_{2}$ such that for all $t \in\left[t_{0}, \infty\right)$ :

$$
\|\boldsymbol{F}(\boldsymbol{X}, t)\|^{2}+\|\boldsymbol{G}(\boldsymbol{X}, t)\|^{2}+\sum_{\ell=1}^{m_{2}} \int_{\left|\xi_{\ell}\right|<\varepsilon_{\ell}}\left\|\boldsymbol{\Phi}^{(\ell)}\left(\boldsymbol{X}, t, \xi_{\ell}\right)\right\|^{2} \nu_{\ell}\left(d \xi_{\ell}\right) \leq L_{2}\left(1+\|\boldsymbol{X}\|^{2}\right) .
$$

\subsection{Definition of a.s. stability}

The following definition is based on (Applebaum, 2009) and (Khalil, 2002).

Definition 2.1: The trial solution of (1) is said to be

(1) almost surely globally practically stable if for $\epsilon \in(0,1)$, there exists a class $\mathcal{K}_{\infty}$-function $\alpha$ such that

$$
\mathbb{P}\left\{\|\boldsymbol{X}(t)\|<\alpha\left(\left\|\boldsymbol{X}_{0}\right\|\right)+\epsilon_{0}\right\} \geq 1-\epsilon
$$

for all $t \in\left[t_{0}, \infty\right)$ and $\boldsymbol{X}_{0} \in \mathbb{R}^{n}$, where $\epsilon_{0}$ is a nonnegative constant.

(2) almost surely globally practically $\mathcal{K}_{\infty}$-exponentially stable if it is almost surely globally practically stable and

$$
\|\boldsymbol{X}(t)\| \leq \alpha\left(\left\|\boldsymbol{X}\left(t_{0}\right)\right\|\right) e^{-\epsilon\left(t-t_{0}\right)}+\epsilon_{0}, \text { a.s. }
$$

for all $\boldsymbol{X}_{0} \in \mathbb{R}^{n}$, where $\alpha$ is a class $\mathcal{K}_{\infty}$-function, $\epsilon$ is a positive constant, and $\epsilon_{0}$ is a nonnegative constant.

If $\epsilon_{0}=0$, "practically" is dropped from the above statements. Moreover, if $\alpha\left(\left\|\boldsymbol{X}\left(t_{0}\right)\right\|\right) \equiv c\left\|\boldsymbol{X}\left(t_{0}\right)\right\|$ with $c$ being a positive constant, then " $\mathcal{K}_{\infty}$ " is dropped from the above statements.

\subsection{A Lyapunov-type theorem}

The following theorem gives sufficient conditions on global well-posedness and stability of nonlinear $\operatorname{SDE}(1)$.

Theorem 2.1: Under Assumption 2.1, assume further that there exists a function $U \in C^{2}\left(\mathbb{R}^{n} \times\right.$ $\left.\left[t_{0}, \infty\right)\right)$ called a Lyapunov function; class $\mathcal{K}_{\infty}$-functions $\alpha_{1}($.$) and \alpha_{2}($.$) ; and an integer p \geq 2$ such that $\forall(\boldsymbol{X}, t) \in \mathbb{R}^{n} \times\left[t_{0}, \infty\right)$ :

$$
\alpha_{1}\left(\|\boldsymbol{X}\|^{p}\right) \leq U(\boldsymbol{X}, t) \leq \alpha_{2}\left(\|\boldsymbol{X}\|^{p}\right) .
$$

1) [Well-posedness] Suppose that $\mathcal{L} U(\boldsymbol{X}, t)$ satisfies

$$
\mathcal{L} U(\boldsymbol{X}, t) \leq c_{3}(1+U(\boldsymbol{X}, t)), \boldsymbol{X} \in V, t \in\left[t_{0}, \infty\right),
$$

where $c_{3}$ is a nonnegative constant. Then the system (1) has a unique solution for each $\boldsymbol{X}_{0} \in \mathbb{R}^{n}$. 2) [a.s. stability] Suppose that there exists a positive constant $c_{4}$ such that $\forall(\boldsymbol{X}, t) \in V \times\left[t_{0}, \infty\right)$ :

$$
\mathcal{L} U(\boldsymbol{X}, t) \leq-c_{4} U(\boldsymbol{X}, t)+c_{0}
$$

If the constant $c_{0}=0$, the equilibrium $\boldsymbol{X} \equiv 0$ is a.s. globally stable. If $c_{0}>0$, the equilibrium $\boldsymbol{X} \equiv 0$ is a.s. globally practically stable.

3) [a.s. exponential stability] Suppose further that $\alpha_{2}\left(\left\|\boldsymbol{X}+\boldsymbol{\Phi}^{\ell}\right\|^{p}\right) \leq \alpha_{21}\left(\|\boldsymbol{X}\|^{p}\right)+\alpha_{22}\left(\left\|\boldsymbol{\Phi}^{\ell}\right\|^{p}\right)$, where $\alpha_{21}\left(\|\boldsymbol{X}\|^{p}\right)$ and $\alpha_{22}\left(\left\|\boldsymbol{\Phi}^{\ell}\right\|^{p}\right)$ are some class $\mathcal{K}_{\infty}$-functions, and the function $\alpha_{22}\left(\left\|\boldsymbol{\Phi}^{\ell}\right\|^{p}\right)$ satisfies

$$
\sup _{\boldsymbol{X} \in \mathbb{E}} \int_{\left|\xi_{\ell}\right|<\varepsilon_{\ell}} e^{\left\|\boldsymbol{\Phi}^{\ell}\left(\boldsymbol{X}, t, \xi_{\ell}\right)\right\|_{\nu}}\left(d \xi_{\ell}\right)<\infty \text { a.s. }
$$

for all bounded sets $\mathbb{E}$. If the constant $c_{0}=0$, the equilibrium $\boldsymbol{X} \equiv 0$ is a.s. globally $\mathcal{K}_{\infty}$ exponentially stable. If $c_{0}>0$, the equilibrium $\boldsymbol{X} \equiv 0$ is a.s. globally practically $\mathcal{K}_{\infty}$-exponentially 
stable. Moreover,

$$
\|\boldsymbol{X}(t)\|^{p} \leq \alpha_{1}^{-1}\left[\alpha_{2}\left(\left\|\boldsymbol{X}_{0}\right\|^{p}\right) e^{-c_{4}\left(t-t_{0}\right)}+\frac{c_{0}}{c_{4}}\right] \text { a.s. }
$$

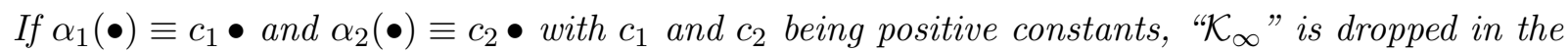
above statements.

Proof. See Appendix A.

\section{Remark 2.1:}

- Only local conditions (2) and (3) are needed. These conditions ensure existence and uniqueness of a local solution to (1). This local solution is then extended to a global solution by the conditions (6) and (7).

- The inequality (10) is equivalent to (5) by taking the power $\frac{1}{p}$ both sides of (10) and applying $(|a|+|b|)^{\ell} \leq 2^{\ell}\left(|a|^{\ell}+|b|^{\ell}\right)$ for all $(a, b) \in \mathbb{R}^{2}$ and $\ell>0$.

- Although conditions imposed in Theorem 2.1 are of the form similar to those for deterministic systems, see e.g., (Khalil, 2002, Chapter 4), the infinite generator $\mathcal{L} U$ has to be used instead of the normal time derivative of $U$. This makes Theorem 2.1 easily accessible to those who are familiar to Lyapunov's direct method for control design and stability analysis.

\section{Stochastic control of magnetic bearings}

\subsection{Mathematical model and control objective}

Since the dynamics of a magnetic bearing system in the $y$-direction is the same as that in the $x$-direction, we only consider its mathematical model in the $x$-direction as follows, see (Smith \& Weldon, 1995):

$$
m \ddot{x}=\sum_{j=1}^{2} F_{i}\left(x, I_{i}\right)+F_{0 x},
$$

where $x(t), \dot{x}(t)$ and $\ddot{x}(t)$ represent the rotor disk position, velocity, and acceleration, respectively, along the $x$-direction; $m$ represents the mass of the rotor; $F_{i}\left(x, I_{i}\right), i=1,2$ denote the forces produced by each stator electromagnetic circuit; $I_{i}, i=1,2$ represent the current in each stator coil; $F_{0 x}$ is the external disturbance force. The forces $F_{i}\left(x, I_{i}\right)$ and $F_{0 x}$ are detailed in what follows.

Under a common assumption that the applied magnetic forces are only dependent on the direction of major motion and the measured current in winding of the coil (for example $F_{1}\left(x, I_{1}\right)$ only depends on $x$ and $I_{1}$ ), the flux linkage model is used to compute the model for the force produced by the electrical phases as follows (Woodson \& Melcher, 1968):

$$
F_{i}\left(x, I_{i}\right)=\frac{\partial}{\partial x} \int_{0}^{I_{i}} \lambda_{i}\left(x, I_{i}\right) d I_{i}, i=1,2
$$

where $\lambda_{i}\left(x, I_{i}\right)$ represents the flux linkage model. If fringing and leakage are neglected and the magnetic circuit is assumed to be linear, the following flux linkage model is often utilized to complete the electromechanical dynamic system description, see (Smith \& Weldon, 1995):

$$
\lambda_{i}\left(x, I_{i}\right)=L_{i}(x) I_{i}, \quad i=1,2,
$$

with

$$
L_{i}(x)=\frac{L_{0}}{2\left(g_{0 x}+(-1)^{i} x\right)+L_{1}}, i=1,2,
$$

where $L_{0}$ and $L_{1}$ are positive constant parameters which depend on the number of stator coil turns, permeability of the material, air, cross-sectional area of the electromagnet, etc., and $g_{0 x}$ is a constant kinematic quantity, i.e. the gap between the rotor and the stator when the rotor is in its neutral position. The flux linkage model given by (13) can now be used to calculate $F_{i}\left(x, I_{i}\right), i=1,2$ 
in (12) explicitly as follows

$$
F_{i}\left(x, I_{i}\right)=\frac{(-1)^{(i+1)} L_{0} I_{i}^{2}}{\left(2\left(g_{0 x}+(-1)^{i} x\right)+L_{1}\right)^{2}}, i=1,2 .
$$

To cover various external disturbance forces in practice, we consider $F_{0 x}$ of the following form:

$$
F_{0 x}=a_{1} f_{1}(x)+a_{2},
$$

where the functions $f_{1}(x)$ is a continuous function of $x$, and is bounded if its argument $x$ is bounded. We use a Lévy process to describe each parameter $a_{i}, i=1,2$ as discussed in Section 1 as follows:

$$
a_{i}=\theta_{i}+\delta_{i} \frac{d w_{i}(t)}{d t}+\gamma_{i} \int_{\left|\xi_{i}\right|<\varepsilon_{i}} \frac{\widetilde{N}_{i}\left(d t, d \xi_{i}\right)}{d t},
$$

where $\theta_{i}, \delta_{i}$, and $\gamma_{i}$ with $i=1,2$ are unknown but bounded parameters and the constant $\varepsilon_{i} \in(0, \infty]$; and $w_{i}(t)$ is a standard Wiener process. The compensated Poisson measure $\widetilde{N}_{i}\left(d t, d \xi_{i}\right)$ is given by $\widetilde{N}_{i}\left(d t, d \xi_{i}\right)=N_{i}\left(d t, d \xi_{i}\right)-d t \nu_{i}\left(d \xi_{i}\right)$, where $N_{i}\left(d t, d \xi_{i}\right)$ is a Poisson measure and $\nu_{i}\left(d \xi_{i}\right)$ is a intensity measure, see also Section 2.1. Substituting (16), where $a_{i}$ are given in (17), into (11) results in the stochastic model of the magnetic bearing system:

$$
\begin{aligned}
& d x_{1}=x_{2} d t \\
& d x_{2}=\frac{1}{m}\left[\left(\sum_{i=1}^{2} F_{i}\left(x_{1}, I_{i}\right)+\sum_{i=1}^{2} \theta_{i} \Phi_{i}\right) d t+\sum_{i=1}^{2} \delta_{i} \Phi_{i} d w_{i}(t)+\sum_{i=1}^{2} \gamma_{i} \Phi_{i} \int_{\left|\xi_{i}\right|<\varepsilon_{i}} \widetilde{N}_{i}\left(d t, d \xi_{i}\right)\right],
\end{aligned}
$$

where $x_{1}=x, x_{2}=\dot{x}$, and

$$
\Phi_{1}=f_{1}\left(x_{1}\right), \Phi_{2}=1 .
$$

Before stating the control objective, we make the following assumptions, which are reasonable in practice.

Assumption 3.1: All the following conditions hold almost surely:

(1) From its neutral position, the rotor can displace in the interval $\left[-\underline{g}_{0 x}, \bar{g}_{0 x}\right]$ along the $x$-axis. The constants $\underline{g}_{0 x}, \bar{g}_{0 x}$ are strictly positive such that $\underline{g}_{0 x} \vee \bar{g}_{0 x} \leq g_{0 x}$.

(2) The reference trajectory $x_{1 d}(t)$ is feasible, i.e., there exist strictly positive constants $\underline{C}_{1 x}^{M}<\underline{g}_{0 x}$ and $\bar{C}_{1 x}^{M}<\bar{g}_{0 x}$ such that

$$
-\left(\underline{g}_{0 x}-\underline{C}_{1 x}^{M}\right) \leq x_{1 d}(t) \leq\left(\bar{g}_{0 x}-\bar{C}_{1 x}^{M}\right) .
$$

Moreover, the time derivatives $\dot{x}_{1 d}(t)$ and $\ddot{x}_{1 d}(t)$ are bounded.

(3) The initial tracking error is feasible, i.e., there exist strictly positive constants $\underline{C}_{0 x}<\underline{C}_{1 x}^{M}$ and $\bar{C}_{0 x}<\bar{C}_{1 x}^{M}$ such that at the initial time $t_{0} \geq 0$, the following conditions hold

$$
-\underline{C}_{0 x} \leq x_{1}\left(t_{0}\right)-x_{1 d}\left(t_{0}\right) \leq \bar{C}_{0 x},
$$

where $x_{1 d}\left(t_{0}\right)$ is the initial value of $x_{1 d}(t)$ at $t=t_{0} \geq 0$.

(4) All the parameters $\theta_{i}(t), \delta_{i}(t), \gamma_{i}(t)$, and $\int_{\left|\xi_{i}\right|<\epsilon_{i}} \nu_{i}\left(d \xi_{i}\right), i=1,2$ are bounded, i.e., there exist nonnegative constants $\bar{\theta}_{i}, \bar{\delta}_{i}, \bar{\gamma}_{i}$, and $\varpi_{i}$ such that

$$
\begin{aligned}
& \sup _{t \in\left[t_{0}, \infty\right)} \theta_{i}^{2 k}(t) \leq \bar{\theta}_{i}, \sup _{t \in\left[t_{0}, \infty\right)} \delta_{i}^{2 k}(t) \leq \bar{\delta}_{i}, \\
& \sup _{t \in\left[t_{0}, \infty\right)} \gamma_{i}^{2 k}(t) \leq \bar{\gamma}_{i}, \sup _{t \in\left[t_{0}, \infty\right)}\left(\int_{\left|\xi_{i}\right|<\epsilon_{i}} \nu_{i}\left(d \xi_{i}\right)\right)^{2 k} \leq \varpi^{2 k}:=\bar{\varpi}_{i},
\end{aligned}
$$

where $k$ is an integer larger than 1.

Control Objective 3.1: Under Assumption 3.1, design the control inputs $I_{i}, i=1,2$ that force the output $x_{1}(t)$, of the magnetic bearing system defined in (18) to track the reference trajectory 
$x_{1 d}(t)$, such that

(1) The output tracking error are within a pre-specified and feasible range almost surely, i.e.,

$$
-\underline{C}_{1 x} \leq x_{1}(t)-x_{1 d}(t) \leq \bar{C}_{1 x}, \text { a.s. }
$$

for all $t \geq t_{0} \geq 0$, where $\underline{C}_{1 x}$ and $\bar{C}_{1 x}$ are strictly positive constants that satisfy the following feasibility conditions

$$
\underline{C}_{0 x}<\underline{C}_{1 x} \leq \underline{C}_{1 x}^{M}, \quad \bar{C}_{0 x}<\bar{C}_{1 x} \leq \bar{C}_{1 x}^{M}, \text { a.s. }
$$

(2) Almost surely exponential tracking is achieved, i.e.

$$
\left|x_{1}(t)-x_{1 d}(t)\right| \leq \varphi_{1}\left(x_{1}\left(t_{0}\right), x_{2}\left(t_{0}\right)\right) e^{-c_{11}\left(t-t_{0}\right)}+c_{10}, \text { a.s. }
$$

where $\varphi_{1}$ is a class $\mathcal{K}$-function, $c_{11}$ is a positive constant, and $c_{10}$ is a nonnegative constant such that $c_{10} \leq \underline{C}_{1 x} \wedge \bar{C}_{1 x}$.

(3) All signals of the closed loop system are bounded.

\subsection{Preliminaries}

As the control objective imposes on both symmetric and asymmetric constraints on the tracking error $x_{1}(t)-x_{1 d}(t)$, see $(23)$, we first need a suitable smooth unbounded and increasing function, which will be embedded in our Lyapunov function in the sequel.

Definition 3.1: Let $a$ and $b$ be constants such that $a<0<b$, a function $\Psi(x, a, b)$ is said to be $a$ smooth unbounded and increasing function on the interval $[a, b]$ if

$$
\begin{aligned}
& \text { 1) } x=0 \Leftrightarrow \Psi(x, a, b)=0, \\
& \text { 2) } \lim _{x \rightarrow a^{+}} \Psi(x, a, b)=-\infty, \lim _{x \rightarrow b^{-}} \Psi(x, a, b)=\infty, \\
& \text { 3) } \Psi(x, a, b) \text { is smooth for all } x \in(a, b), \\
& \text { 4) } \Psi^{\prime}(x, a, b)>0 \text { for all } x \in(a, b),
\end{aligned}
$$

where $\Psi^{\prime}(x, a, b)=\frac{\partial \Psi(x, a, b)}{\partial x}$.

If $a=-b$, it is then easy to find many smooth unbounded and increasing functions such as $\tanh ^{-1}\left(-\frac{1}{a} x\right)$. If $a \neq-b$, construction of smooth unbounded and increasing functions is given in the following lemma:

Lemma 3.1: For given constants $a$ and $b$ such that $a<0<b$ and $\frac{a+b}{b-a} \neq 2 n+1$ for all $n=$ $0, \pm 1, \pm 2, \cdots$, the scalar function $\Psi(x, a, b)$ defined by

$$
\Psi(x, a, b)=\tan \left[\frac{\pi}{b-a}\left(x-\frac{a+b}{2}\right)\right]+\tan \left[\frac{\pi}{b-a} \frac{a+b}{2}\right]
$$

is a smooth unbounded and increasing function of $x$ on the interval $[a, b]$. Moreover, $\Psi^{\prime}(x, a, b) \geq$ $\frac{\pi}{b-a}, \forall x \in \mathbb{R}$.

Proof. The proof follows by a direct verification of all properties of smooth unbounded and increasing functions listed in (26). Lemma 3.1 provides a much simpler construction of smooth unbounded and increasing functions than Lemma 2.2 in (Do, 2010). A plot of smooth unbounded and increasing function with $a=-2$ and $b=4$ is given in Fig. 1 .

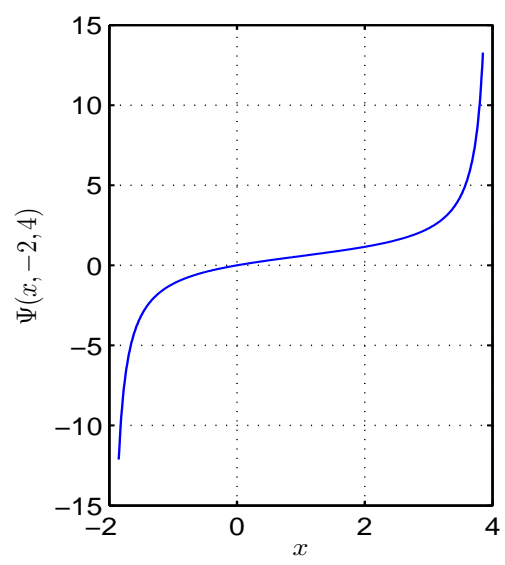

Figure 1.: A plot of $\Psi(x,-2,4)$. 


\subsection{Coordinate transformation}

To transform the tracking control objective with constraints to a stabilization problem, we introduce the coordinate transformation

$$
z_{1}=\Psi\left(x_{1 e}, a, b\right),
$$

where $x_{1 e}=x_{1}-x_{d}$ is the output tracking error. The function $\Psi\left(x_{1 e}, a, b\right)$ is chosen according to Lemma 3.1. The constants $a$ and $b$, are chosen such that

$$
\begin{aligned}
& -\underline{C}_{1 x} \leq a<-\underline{C}_{0 x}, \bar{C}_{0 x}<b \leq \bar{C}_{1 x}, \\
& \frac{a+b}{b-a} \neq 2 n+1, \forall n=0, \pm 1, \pm 2, \cdots
\end{aligned}
$$

Applying the Itô formula, see (Applebaum, 2009), to (28) along the solutions of (18) gives

$$
\begin{aligned}
& d z_{1}=\Psi^{\prime}\left(x_{1 e}, a, b\right)\left(x_{2}-\dot{x}_{d}\right) d t \\
& d x_{2}=\frac{1}{m}\left[\left(\sum_{i=1}^{2} F_{i}\left(x_{1}, I_{i}\right)+\sum_{i=1}^{2} \theta_{i} \Phi_{i}\right) d t+\sum_{i=1}^{2} \delta_{i} \Phi_{i} d w_{i}(t)+\sum_{i=1}^{2} \gamma_{i} \Phi_{i} \int_{\left|\xi_{i}\right|<\varepsilon_{i}} \widetilde{N}_{i}\left(d t, d \xi_{i}\right)\right] .
\end{aligned}
$$

Thus, the control objective now is to design $I_{i}, i=1,2$ such that they stabilize (30) at the origin almost surely.

\subsection{Control design}

The control design consists of two steps by applying the ingredients developed in Section 2 and the backstepping method (Krstic, Kanellakopoulos, \& Kokotovic, 1995), which are detailed below.

\subsubsection{Step 1}

Define

$$
z_{2}=x_{2}-\alpha_{1}
$$

where $\alpha_{1}$ is a virtual control of $x_{2}$. To design $\alpha_{1}$, we consider the following Lyapunov function candidate

$$
U_{1}=\frac{1}{2 k} z_{1}^{2 k}
$$

where $k$ is a positive integer to be determined. The above choice of $U_{1}$ is motivated by the work in (Do, 2015) to handle the Hessian term in the infinite generator $\mathcal{L} U_{2}$, which will appear in the second step. Applying the Itô formula to $U_{1}$ along the solutions of the first equation of (30) and (31) yields

$$
\mathcal{L} U_{1}=z_{1}^{2 k-1} \Psi^{\prime}\left(z_{2}+\alpha_{1}-\dot{x}_{d}\right),
$$

where we have dropped the arguments $\left(x_{1 e}, a, b\right)$ of $\Psi^{\prime}$ for clarity. From (33), we choose $\alpha_{1}$ as

$$
\alpha_{1}=-k_{11} z_{1}-k_{12}\left(\Psi^{\prime}\right)^{2 k-1} z_{1}+\dot{x}_{d},
$$

where $k_{11}$ and $k_{12}$ are positive constants to be chosen later. It is noted that $\alpha_{1}$ is a function of $\left(x_{1}, x_{d}, \dot{x}_{d}\right)$. Substituting (34) into (33) gives

$$
\mathcal{L} U_{1}=-\left[k_{11} \Psi^{\prime}+k_{12}\left(\Psi^{\prime}\right)^{2 k}\right] z_{1}^{2 k}+\Psi^{\prime} z_{1}^{2 k-1} z_{2} .
$$




\subsubsection{Step 2}

To design the controls $I_{1}$ and $I_{2}$, we consider the following Lyapunov function candidate

$$
U_{2}=U_{1}+\frac{m}{2 k} z_{2}^{2 k} \text {. }
$$

Applying the Itô formula to $U_{2}$ along the solutions of the second equation of (30) and using (35) results in

$$
\begin{aligned}
\mathcal{L} U_{2} & =-\left[k_{11} \Psi^{\prime}+k_{12}\left(\Psi^{\prime}\right)^{2 k}\right] z_{1}^{2 k}+\Psi^{\prime} z_{1}^{2 k-1} z_{2} \\
& +z_{2}^{2 k-1}\left(\sum_{i=1}^{2} F_{i}\left(x_{1}, I_{i}\right)+\sum_{i=1}^{2} \theta_{i} \Phi_{i}-m\left(\frac{\partial \alpha_{1}}{\partial x_{1}} x_{2}+\frac{\partial \alpha_{1}}{\partial x_{d}} \dot{x}_{d}+\frac{\partial \alpha_{1}}{\partial \dot{x}_{d}} \ddot{x}_{d}\right)\right)+\frac{2 k-1}{2} z_{2}^{2 k-2} \sum_{i=1}^{2} \delta_{i}^{2} \Phi_{i}^{2} \\
& +m \sum_{i=1}^{2} \int_{\left|\xi_{i}\right|<\varepsilon_{i}}\left[\frac{1}{2 k}\left(z_{2}+\gamma_{i} \Phi_{i}\right)^{2 k}-\frac{1}{2 k} z_{2}^{2 k}-z_{2}^{2 k-1} \gamma_{i} \Phi_{i}\right] \nu_{i}\left(d \xi_{i}\right) .
\end{aligned}
$$

We now need to find the upper-bound of the right hand-side of (37). Using the inequalities $(a+$ $b)^{2 k} \leq 2^{2 k-1}\left(a^{2 k}+b^{2 k}\right)$ and $a b \leq \frac{\epsilon^{p}}{p}|a|^{p}+\frac{1}{q \epsilon^{q}}|b|^{q}$ with $\epsilon>0, p>1, q>1, \frac{1}{p}+\frac{1}{q}=1$ for all $(a, b) \in \mathbb{R}^{2}$, and the expression of $\Phi_{i}, i=1,2$ in (19), we can calculate:

$$
\begin{aligned}
& \Psi^{\prime} z_{1}^{2 k-1} z_{2} \leq\left(\Psi^{\prime}\right)^{2 k} \epsilon_{10}^{\frac{2 k}{2 k-1}} z_{1}^{2 k}+\frac{1}{2 k \epsilon_{10}^{2 k}} z_{2}^{2 k}, \\
& z_{2}^{2 k-1} \theta_{1} \Phi_{1} \leq \frac{2 k-1}{2 k} \epsilon_{11}^{\frac{2 k}{2 k-1}} z_{2}^{2 k}+\frac{1}{2 k \epsilon_{11}^{2 k}} \bar{\theta}_{1} \bar{A}, \\
& z_{2}^{2 k-1} \theta_{2} \Phi_{2} \leq \frac{2 k-1}{2 k} \epsilon_{12}^{\frac{2 k}{2 k-1}} z_{2}^{2 k}+\frac{1}{2 k \epsilon_{12}^{2 k}} \bar{\theta}_{2}, \\
& z_{2}^{2 k-1} \delta_{1} \Phi_{1} \leq \frac{2 k-1}{2 k} \epsilon_{11}^{\frac{2 k}{2 k-1}} z_{2}^{2 k}+\frac{1}{2 k \epsilon_{11}^{2 k}} \bar{\delta}_{1} \bar{A}, \\
& z_{2}^{2 k-1} \delta_{2} \leq \frac{2 k-1}{2 k} \epsilon_{12}^{\frac{2 k}{2 k-1}} z_{2}^{2 k}+\frac{1}{2 k \epsilon_{12}^{2 k}} \bar{\delta}_{2}, \\
& m \int_{\left|\xi_{1}\right|<\varepsilon_{1}} \frac{1}{2 k}\left(z_{2}+\gamma_{1} \Phi_{1}\right)^{2 k} \nu_{1}\left(d \xi_{1}\right) \leq \frac{m 2^{2 k-1}}{2 k} \varpi_{1} z_{2}^{2 k}+\frac{m 2^{2 k-1}}{2 k} \bar{\gamma}_{1} \bar{A} \varpi_{1}, \\
& m \int_{\left|\xi_{1}\right|<\varepsilon_{1}} z_{2}^{2 k-1} \gamma_{1} \Phi_{1} \nu_{1}\left(d \xi_{1}\right) \leq \frac{m 2^{2 k-1}}{2 k} \epsilon_{15}^{\frac{2 k}{2 k-1}} z_{2}^{2 k}+\frac{m}{2 k \epsilon_{15}^{2 k}} \bar{\gamma}_{1} \bar{A} \bar{\varpi}_{1}, \\
& m \int_{\left|\xi_{2}\right|<\varepsilon_{2}} \frac{1}{2 k}\left(z_{2}+\gamma_{2} \Phi_{2}\right)^{2 k} \nu_{2}\left(d \xi_{2}\right) \leq \frac{m 2^{2 k-1}}{2 k} \varpi_{2} z_{2}^{2 k}+\frac{m 2^{2 k-1}}{2 k} \bar{\gamma}_{2} \varpi_{2}, \\
& m \int_{\left|\xi_{2}\right|<\varepsilon_{2}} z_{2}^{2 k-1} \gamma_{2} \Phi_{2} \nu_{2}\left(d \xi_{2}\right) \leq \frac{m 2^{2 k-1}}{2 k} \epsilon_{16}^{\frac{2 k}{2 k-1}} z_{2}^{2 k}+\frac{m}{2 k \epsilon_{16}^{2 k}} \bar{\gamma}_{2} \bar{\varpi}_{2}, \\
& \cdots \bar{\varpi}_{2},
\end{aligned}
$$

where $\epsilon_{1 i}, i=0, \cdots, 6$ are positive constants to be chosen later; $\bar{\delta}_{i}, \bar{\gamma}_{i}, \bar{\varpi}_{i}, i=1,2$ are defined in (22); $\bar{A}=\left(\sup _{x \in\left[a-\left|x_{d}\right|, b+\left|x_{d}\right|\right]}\left|f_{1}(x)\right|\right)^{2 k}$ with a note that we have used (27) and (28) to obtain $x=\arctan \left(z_{1}-\tan \left(\frac{\pi}{b-a} \frac{a+b}{2}\right)\right) \frac{b-a}{\pi}+\frac{a+b}{2}+x_{d}$. Substituting (38) into (37) results in

$$
\begin{aligned}
\mathcal{L} U_{2} \leq & -\left[k_{11} \Psi^{\prime}+\left(k_{12}-\epsilon_{10}^{\frac{2 k}{2 k-1}}\right)\left(\Psi^{\prime}\right)^{2 k}\right] z_{1}^{2 k}+z_{2}^{2 k-1}\left(\sum_{i=1}^{2} F_{i}\left(x_{1}, I_{i}\right)\right. \\
& \left.-m\left(\frac{\partial \alpha_{1}}{\partial x_{1}} x_{2}+\frac{\partial \alpha_{1}}{\partial x_{d}} \dot{x}_{d}+\frac{\partial \alpha_{1}}{\partial \dot{x}_{d}} \ddot{x}_{d}\right)+c_{1} z_{2}\right)+c_{0},
\end{aligned}
$$

where

$$
\begin{aligned}
c_{1}= & \frac{1}{2 k \epsilon_{10}^{2 k}}+\frac{2 k-1}{2 k} \epsilon_{11}^{\frac{2 k}{2 k-1}}+\frac{2 k-1}{2 k} \epsilon_{12}^{\frac{2 k}{2 k-1}}+\frac{2 k-1}{2 k} \epsilon_{13}^{\frac{2 k}{2 k-1}}+\frac{2 k-1}{2 k} \epsilon_{14}^{\frac{2 k}{2 k-1}}+\frac{m 2^{2 k-1}}{2 k} \varpi_{1}+\frac{m 2^{2 k-1}}{2 k} \epsilon_{15}^{\frac{2 k}{2 k-1}}+\frac{m 2^{2 k-1}}{2 k} \varpi_{2} \\
& +\frac{m 2^{2 k-1}}{2 k} \epsilon_{16}^{2 k-1} \\
c_{0}= & \frac{1}{2 k \epsilon_{11}^{2 k}} \overline{1}_{1} \bar{A}+\frac{1}{2 k \epsilon_{12}^{2 k}} \bar{\theta}_{2}+\frac{1}{2 k \epsilon_{13}^{2 k}} \bar{\delta}_{1} \bar{A}+\frac{1}{2 k \epsilon_{14}^{2 k}} \bar{\delta}_{2}+\frac{m 2^{2 k-1}}{2 k} \bar{\gamma}_{1} \bar{A} \varpi_{1}+\frac{m}{2 k \epsilon_{15}^{2 k}} \bar{\gamma}_{1} \bar{A} \bar{\varpi}_{1}+\frac{m 2^{2 k-1}}{2 k} \bar{\gamma}_{2} \varpi_{2} \\
& +\frac{m}{2 k \epsilon_{16}^{2 k}} \bar{\gamma}_{2} \bar{\varpi}_{2} .
\end{aligned}
$$

From (39), we choose the controls $I_{1}$ and $I_{2}$ such that

$$
\begin{aligned}
\sum_{i=1}^{2} F_{i}\left(x_{1}, I_{i}\right) & =-k_{2} z_{2}+m\left(\frac{\partial \alpha_{1}}{\partial x_{1}} x_{2}+\frac{\partial \alpha_{1}}{\partial x_{d}} \dot{x}_{d}+\frac{\partial \alpha_{1}}{\partial \dot{x}_{d}} \ddot{x}_{d}\right)-c_{1} z_{2} \\
:=\Delta &
\end{aligned}
$$


where $k_{2}$ is a positive constant to be chosen later. We now write (41) with (15) as follows

$$
\chi_{1}^{2}\left(x_{1}\right) I_{1}^{2}-\chi_{2}^{2}\left(x_{1}\right) I_{2}^{2}=\Delta,
$$

where $\Delta$ is defined in (41), and

$$
\begin{aligned}
& \chi_{1}\left(x_{1}\right)=\frac{\sqrt{L_{0}}}{2\left(g_{0 x}-x_{1}\right)+L_{1}}, \\
& \chi_{2}\left(x_{1}\right)=\frac{\sqrt{L_{0}}}{2\left(g_{0 x}+x_{1}\right)+L_{1}} .
\end{aligned}
$$

To solve (42) for $I_{1}$ and $I_{2}$, we write (42) as follows

$$
\left(\chi_{1}\left(x_{1}\right) I_{1}-\chi_{2}\left(x_{1}\right) I_{2}\right)\left(\chi_{1}\left(x_{1}\right) I_{1}+\chi_{2}\left(x_{1}\right) I_{2}\right)=\frac{\Delta}{\left(\Delta^{\ell}+\gamma_{0}^{\ell}\right)^{\frac{1}{2 \ell}}}\left(\Delta^{\ell}+\gamma_{0}^{\ell}\right)^{\frac{1}{2 \ell}},
$$

where $\gamma_{0}$ is a small positive constant, and $\ell$ is a positive even number. Thus, we can simply choose

$$
\begin{aligned}
& \chi_{1}\left(x_{1}\right) I_{1}-\chi_{2}\left(x_{1}\right) I_{2}=\left(\Delta^{\ell}+\gamma_{0}^{\ell}\right)^{\frac{1}{2 \ell}}, \\
& \chi_{1}\left(x_{1}\right) I_{1}+\chi_{2}\left(x_{1}\right) I_{2}=\frac{\Delta}{\left(\Delta^{\ell}+\gamma_{0}^{\ell}\right)^{\frac{1}{2 \ell}}},
\end{aligned}
$$

which gives

$$
\begin{aligned}
& I_{1}=\frac{1}{2 \chi_{1}\left(x_{1}\right)}\left(\frac{\Delta}{\left(\Delta^{\ell}+\gamma_{0}^{\ell}\right)^{\frac{1}{2 \ell}}}+\left(\Delta^{\ell}+\gamma_{0}^{\ell}\right)^{\frac{1}{2 \ell}}\right), \\
& I_{2}=\frac{1}{2 \chi_{2}\left(x_{1}\right)}\left(\frac{\Delta}{\left(\Delta^{\ell}+\gamma_{0}^{\ell}\right)^{\frac{1}{2 \ell}}}-\left(\Delta^{\ell}+\gamma_{0}^{\ell}\right)^{\frac{1}{2 \ell}}\right),
\end{aligned}
$$

Remark 3.1: The choice of the controls $I_{1}$ and $I_{2}$ as in (46) is not unique as we can specify alternative terms for $\left(\Delta^{\ell}+\gamma_{0}^{\ell}\right)^{\frac{1}{2 \ell}}$ in (44). Other choices like the ones in (de Queiroz 8 Dawson, 1996; de Queiroz et al., 1998; Do, 2010) are possible. The controls $I_{1}$ and $I_{2}$ in (46) are smooth and their magnitude is proportional to $\sqrt{|\Delta|}$, which makes the controls $I_{1}$ and $I_{2}$ behave very similar to ideal switching controls such as if $\triangle \geq 0$ then $I_{1}=\frac{1}{\chi_{1}\left(x_{1}\right)} \sqrt{\triangle}$ and $I_{2}=0$, else if $\triangle<0$ then $I_{1}=0$ and $I_{2}=\frac{-1}{\chi_{2}\left(x_{1}\right)} \sqrt{-\triangle}$ when $|\triangle|$ dominates $\gamma_{0}$. Indeed, if $\gamma_{0}$ is set to zero, the controls $I_{1}$ and $I_{2}$ in (46) become

$$
\begin{aligned}
& I_{1}=\frac{1}{2 \chi_{1}\left(x_{1}\right)}\left(\frac{\Delta}{\sqrt{|\Delta|}}+\sqrt{|\Delta|}\right), \\
& I_{2}=\frac{1}{2 \chi_{2}\left(x_{1}\right)}\left(\frac{\Delta}{\sqrt{|\Delta|}}-\sqrt{|\Delta|}\right),
\end{aligned}
$$

which behave exactly the same as the aforementioned ideal switching controls. However, the controls $I_{1}$ and $I_{2}$ in (47) are only continuous instead of being smooth as those in (46). The tradeoff is that the controls $I_{1}$ and $I_{2}$ in (46) require a small quantity of currents when $\Delta=0$ due to nonzero $\gamma_{0}$.

Substituting (41) into (39) gives

$$
\mathcal{L} U_{2} \leq-\left[k_{11} \Psi^{\prime}+\left(k_{12}-\epsilon_{10}^{\frac{2 k}{2 k-1}}\right)\left(\Psi^{\prime}\right)^{2 k}\right] z_{1}^{2 k}-k_{2} z_{2}^{2 k}+c_{20}
$$

For a given $\epsilon_{10}$, we first choose $k_{12}$ such that

$$
k_{12} \geq \epsilon_{10}^{\frac{2 k}{2 k-1}}
$$

Using (49) and $\Psi^{\prime} \geq \frac{\pi}{b-a}$, see Lemma 3.1, and definition of $U_{2}$ given in (36), we can write (48) as follows:

$$
\mathcal{L} U_{2} \leq-c U_{2}+c_{0},
$$


where

$$
c=2 k\left[\left(\frac{k_{11} \pi}{b-a}+\left(k_{12}-\epsilon_{30}^{\frac{2 k}{2 k-1}}\right)\left(\frac{\pi}{b-a}\right)^{2 k}\right) \wedge k_{2}\right] .
$$

The control design has been completed. We summarize the main results in the following theorem.

Theorem 3.1: Under Assumption 3.1, the controls $I_{1}$ and $I_{2}$ given in (46) ensure that the closedloop system consisting of (41) and (30) or (18) is well-posed; $x_{1 e}(t) \in(a, b)$ for all $t \geq t_{0}$ almost surely; and $\left|x_{1 e}(t)\right|$ almost surely exponentially converges to $c_{0}^{\star}$ defined by

$$
\begin{aligned}
c_{0}^{\star}= & {\left[\arctan \left(2^{\frac{1}{2 k}}\left[2^{2 k-1} 2 k \frac{c_{0}}{c}\right]^{\frac{1}{2 k}}-\tan \left(\frac{\pi}{b-a} \frac{a+b}{2}\right)\right) \frac{b-a}{\pi}+\frac{a+b}{2}\right] } \\
\vee & {\left[\left|-\arctan \left(2^{\frac{1}{2 k}}\left[2^{2 k-1} 2 k \frac{c_{0}}{c}\right]^{\frac{1}{2 k}}+\tan \left(\frac{\pi}{b-a} \frac{a+b}{2}\right)\right) \frac{b-a}{\pi}+\frac{a+b}{2}\right|\right], }
\end{aligned}
$$

which can be made arbitrarily small choosing large control gains $k_{11}, k_{12}$, and $k_{2}$ because $c$ is given by (51). Control Objective 3.1 is solved by the controls $I_{1}$ and $I_{2}$ given in (46) provided that control gains $k_{11}, k_{12}$, and $k_{2}$ are chosen such that $c_{0}^{\star} \leq c_{10}$, where $c_{10}$ is defined in (25).

Proof. First, applying Theorem 2.1 to (50) with $U_{2}$ given in (36) ensures that the closed-loop system consisting of (41) and (30) or the $x$-subsystem given in (18) is well-posed. Next, applying Theorem 2.1 to (50) with $U_{2}$ given in (36) yields

$$
\begin{aligned}
z_{1}^{2 k}(t)+z_{2}^{2 k}(t) & \leq 2 k\left(\frac{1}{2 k}\left(z_{1}^{2 k}\left(t_{0}\right)+z_{2}^{2 k}\left(t_{0}\right)\right) e^{-c\left(t-t_{0}\right)}+\frac{c_{0}}{c}\right) \text { a.s. } \\
\Rightarrow\left|z_{1}(t)\right|+\left|z_{2}(t)\right| & \leq\left[2^{2 k-1} 2 k\left(\frac{1}{2 k}\left(z_{1}^{2 k}\left(t_{0}\right)+z_{2}^{2 k}\left(t_{0}\right)\right) e^{-c\left(t-t_{0}\right)}+\frac{c_{0}}{c}\right)\right]^{\frac{1}{2 k}} \\
\leq & 2^{\frac{1}{2 k}}\left[2^{2 k-1} 2 k\left(\frac{1}{2 k}\left(z_{1}^{2 k}\left(t_{0}\right)+z_{2}^{2 k}\left(t_{0}\right)\right) e^{-c\left(t-t_{0}\right)}\right]^{\frac{1}{2 k}}+2^{\frac{1}{2 k}}\left[2^{2 k-1} 2 k \frac{c_{0}}{c}\right]^{\frac{1}{2 k}}\right. \text { a.s. }
\end{aligned}
$$

where we have used $\left(\left|z_{1}\right|+\left|z_{2}\right|\right)^{2 k} \leq 2^{2 k-1}\left(z_{1}^{2 k}+z_{2}^{2 k}\right)$ for all $\left(z_{1}, z_{2}\right) \in \mathbb{R}^{2}$ and $(|a|+|b|)^{\frac{1}{2 k}} \leq$ $2^{\frac{1}{2 k}}\left(|a|^{\frac{1}{2 k}}+|b|^{\frac{1}{2 k}}\right)$ for all $(a, b) \in \mathbb{R}^{2}$. The inequality (53) ensures that $z_{1}(t)$ and $z_{2}(t)$ are bounded for all bounded initial values $z_{1}\left(t_{0}\right)$ and $z_{2}\left(t_{0}\right)$, i.e., for all initial values of $x_{1 e}\left(t_{0}\right) \in(a, b)$ and bounded $x_{2}\left(t_{0}\right)$ due to $(28)$. Solving (28) gives

$$
x_{1 e}=\arctan \left(z_{1}-\tan \left(\frac{\pi}{b-a} \frac{a+b}{2}\right)\right) \frac{b-a}{\pi}+\frac{a+b}{2} .
$$

This together with boundedness of $\left(z_{1}(t), z_{2}(t)\right)$ implies that $x_{1 e}(t) \in(a, b)$ for all initial values of $x_{1 e}\left(t_{0}\right) \in(a, b)$ and bounded $x_{2}\left(t_{0}\right)$. Combining (53) and (54) yields

$$
\begin{aligned}
\left|x_{1 e}(t)\right| \leq & {\left[\arctan \left(\left|z_{1}(t)\right|-\tan \left(\frac{\pi}{b-a} \frac{a+b}{2}\right)\right) \frac{b-a}{\pi}+\frac{a+b}{2}\right] } \\
& \vee\left[\left|-\arctan \left(\left|z_{1}(t)\right|+\tan \left(\frac{\pi}{b-a} \frac{a+b}{2}\right)\right) \frac{b-a}{\pi}+\frac{a+b}{2}\right|\right] \text { a.s. }
\end{aligned}
$$

Since $\arctan (\cdot)$ is a class $\mathcal{K}$-function, almost surely exponential convergence of $\left|z_{1}(t)\right|$ to $2^{\frac{1}{2 k}}\left[2^{2 k-1} 2 k \frac{c_{0}}{c}\right]^{\frac{1}{2 k}}$, which is implied from (53), implies that of $x_{1 e}(t)$ to $c_{0}^{\star}$ defined in $(52)$. Finally, it is noted from (43) that $\chi_{1}\left(x_{1}\right)$ and $\chi_{2}\left(x_{1}\right)$ are well-defined because $\underline{g}_{0 x} \vee \bar{g}_{0 x} \leq g_{0 x}$, see Item 1 of Assumption 3.1.

\subsection{Simulations}

The system parameters are taken as $m=2.0[\mathrm{~kg}], g_{0}=10^{-3}[\mathrm{~m}], L_{0}=3.0 \times 10^{-4}[\mathrm{Hm}], L_{1}=$ $1.25 \times 10^{-5}[\mathrm{~m}], R_{1}=R_{2}=1.0[\Omega]$. We simulate a problem of forcing the rotor to track a sinusoidal signal given by $x_{d}(t)=\frac{g_{0}}{2} \sin (0.5 t)$. The initial conditions are

$$
x_{1}(0)=0.6 \times 10^{-3}[\mathrm{~m}], x_{2}(0)=5 \times 10^{-3}[\mathrm{~m} / \mathrm{s}] .
$$

Based on the conditions specified in Assumption 3.1 and Control Objective 3.1, we take $\underline{C}_{1 x}^{M}=$ $\bar{C}_{1 x}^{M}=0.9 g_{0}, \underline{C}_{1 x}=\bar{C}_{1 x}=0.9^{2} g_{0}, \underline{C}_{0 x}=\bar{C}_{0 x}=0.9^{3} g_{0}$. 
For the disturbance force $F_{0 x}$, the function $f_{1}\left(x_{1}\right)$ is taken as $f_{1}\left(x_{1}\right)=\left|x_{1}\right|^{0.723}$ (usually used in calculation of metal cutting force (Stachurski, Midera, \& Kruszýnski, 2012)); the deterministic parameters are taken as $\theta_{1}=200$ and $\theta_{2}=19.6[N]$; the diffuse and jump coefficients $\delta_{i}$ and $\gamma_{i}$ are taken to be $50 \%$ and $30 \%$ of their corresponding $\theta_{i}$. The Poisson measures $d t \nu_{i}\left(d \xi_{i}\right)$ are taken as $d t \nu_{i}\left(d \xi_{i}\right)=\lambda_{i} \psi\left(\xi_{i}\right) d \xi_{i} d t$, where $\lambda_{1}=5$ and $\lambda_{2}=7, \psi\left(\xi_{i}\right)=\frac{1}{\sqrt{2 \pi} \xi_{i}} e^{-\xi_{i}^{2} / 2}$, which is the density function of a lognormal random variable, and $\varepsilon_{1}=\varepsilon_{2}=10^{3}$. Based on Theorem 3.1, the control design gains are chosen as

$$
k_{11}=1, k_{12}=2, k_{2}=8, \gamma_{0 x}=0.15 .
$$

The parameters $a$ and $b$ of the function $\Psi\left(x_{1 e}, a, b\right)$ are chosen as $a=-0.85 g_{0}$ and $b=0.85 g_{0}$.
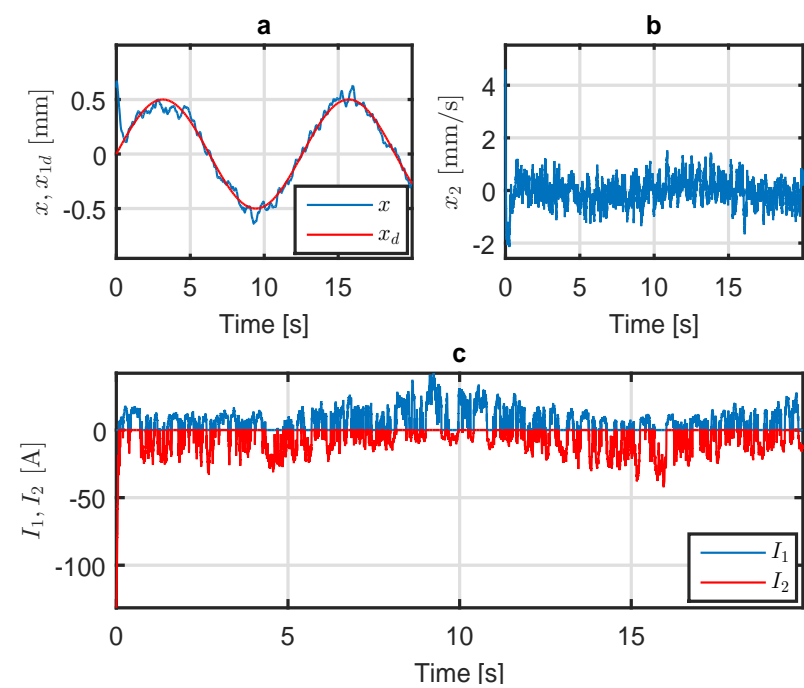

Figure 2.: Results by proposed control design
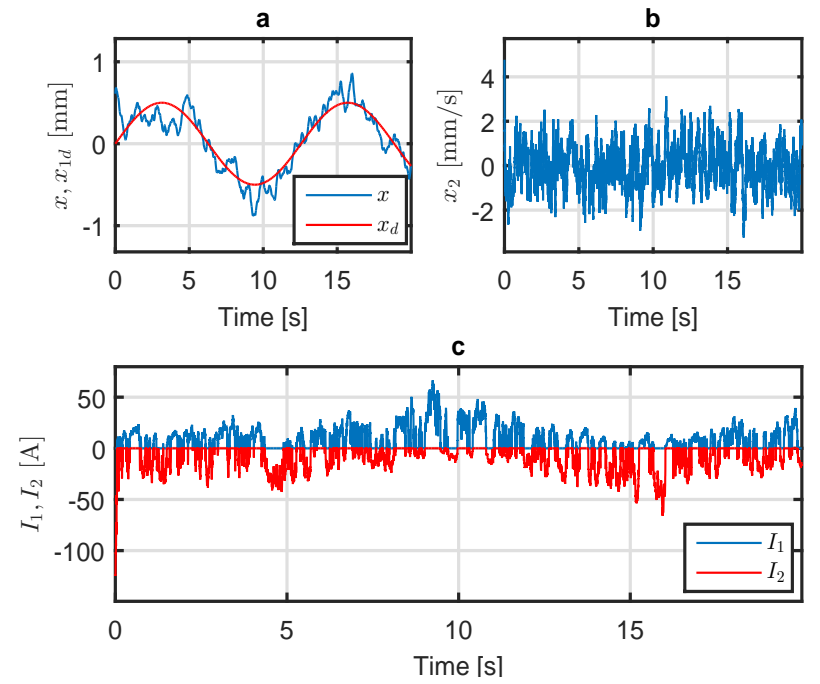

Figure 3.: Results by control design in (Do, 2010)

Simulation results are plotted in Figure 2, where the displacement $x_{1}$ and its reference $x_{d}$ are plotted in Sub-figure 2.a, the velocity $\frac{d x_{1}}{d t}$ is plotted in Sub-figure 2.b, and the control currents $I_{1}$ and $I_{2}$ are plotted in Sub-figure 2.c. It is seen from Sub-figure 2.a that the proposed controls force the rotor displacement to track its reference very well. From Sub-figure 2.c, we can see that the 
control currents $I_{1}$ and $I_{2}$ are fairly large at the beginning. This is because of the fact that the initial position $x_{1}(0)=0.6 \times 10^{-3}[\mathrm{~m}]$ is fairly close to its boundary value $b$, and the initial velocity $x_{2}(0)=5 \times 10^{-3}[\mathrm{~m} / \mathrm{s}]$ pushes the rotor toward the boundary value $b$. Therefore, the control currents must provide sufficiently large control efforts to push the rotor back to prevent it from contacting with the stator. Then at the steady state, the currents $I_{1}$ and $I_{2}$ need to produce sufficient large force to handle the external disturbance force $F_{0 x}$, see (16) and (17) for the expression of this force with the above value of the parameters $\left(\theta_{i}, \delta_{i}, \gamma_{i}\right)$.

Since existing works on control of magnetic bearings only consider deterministic disturbances as discussed in Section 1, it is not possible to run a simulation from these works on the stochastic model of magnetic bearings in this paper. This is because the diffuse and jump terms are simply unbounded in the deterministic sense. Thus, the diffuse and jump terms are first saturated by the magnitude of $\delta_{i}$ and $\gamma_{i}, i=1,2$. Then the control design for magnetic bearings under deterministic loads in (Do, 2010) is implemented, where it is noted that the voltage-dynamics in (Do, 2010) is removed as we are considering magnetic bearings with current driving mode. Simulation results of the control design in (Do, 2010) with diffuse and jump terms being saturated are shown in Figure 3. The information presented in each sub-figure has the same meaning as that in Figure 2. It is seen that deterministic design results in significantly poorer performance in comparison with the stochastic design in this paper. This is due to the fact that the deterministic control design considers the time derivative of the Lyapunov function while the stochastic control design addresses the infinite generator of the Lyapunov function, which has additional Hessian and jump terms (see the last two terms in (37)) compared with its time derivative. Thus, the stochastic control design needs to handle these terms while the deterministic control design does not. These terms actually present in the system. The above analysis explains why the stochastic control design results in better performance than the deterministic one. Note that other controllers those papers such as (de Queiroz \& Dawson, 1996) and (de Queiroz et al., 1998) would give worse results as the hard constraint on the tracking error is not imposed, see (Do, 2010) for a discussion.

\section{Conclusions}

The paper developed a Lyapunov-type theorem to investigate well-posedness and (global practical $\mathcal{K}_{\infty}$ ) exponential stability of SDEs driven by Lévy processes almost surely. Sufficient conditions imposed by the theorem are relatively easy to be verified. The theorem was then applied to design controllers to achieve a.s. practical $\mathcal{K}$-exponential stability of magnetic bearings driven by stochastic diffuse-jumps. Future work is to apply Theorem 2.1 to financial and other engineering systems driven by diffuses and jumps.

\section{Appendix A. Proof of Theorem 2.1}

\section{A.1 Proof of Item 1) of Theorem 2.1}

We first need a lemma on global well-posedness of (1) if the conditions (2) and (3) hold globally.

Lemma A.1: Suppose that the conditions (2) and (3) hold for all $\boldsymbol{X}(t) \in \mathbb{R}^{n}$ and $\boldsymbol{Y}(t) \in \mathbb{R}^{n}$. There exists a unique solution to (1) with the initial condition $\boldsymbol{X}_{0} \in \mathbb{R}^{n}$ almost surely (a.s.) for which $\boldsymbol{X}_{0}$ is $\mathcal{F}_{t_{0}}$-measurable. This solution is adapted and càdlàg.

Proof. See (Applebaum, 2009), Theorem 6.2.3.

We now turn to proof of Item 1) of Theorem 2.1. Let $k_{0}$ be the boundedness of the initial data, 
i.e., $\left\|\boldsymbol{X}_{0}\right\| \leq k_{0}$. For any integer $k>k_{0}$, let us define

$$
\begin{aligned}
& \boldsymbol{F}_{k}(\boldsymbol{X}, t)=\boldsymbol{F}\left(\frac{\|\boldsymbol{X}\| \wedge k}{\|\boldsymbol{X}\|} \boldsymbol{X}, t\right), \\
& \boldsymbol{G}_{k}(\boldsymbol{X}, t)=\boldsymbol{G}\left(\frac{\|\boldsymbol{X}\| \wedge k}{\|\boldsymbol{X}\|} \boldsymbol{X}, t\right), \\
& \boldsymbol{\Phi}_{k}(\boldsymbol{X}, t, \boldsymbol{\xi})=\boldsymbol{\Phi}\left(\frac{\|\boldsymbol{X}\| \wedge k}{\|\boldsymbol{X}\|} \boldsymbol{X}, t, \boldsymbol{\xi}\right),
\end{aligned}
$$

where we let $\frac{\|\boldsymbol{X}\| \wedge k}{\|\boldsymbol{X}\|} \boldsymbol{X}=0$ if $\boldsymbol{X}=0$. Then, it is clear that all the conditions of Lemma A.1 hold for all $\boldsymbol{X} \in \mathbb{R}^{n}$. Thus, Lemma A.1 ensures well-posedness of the solution $\boldsymbol{X}_{k}(t)$ to the SDE:

$$
d \boldsymbol{X}_{k}=\boldsymbol{F}_{k}\left(\boldsymbol{X}_{k}, t\right) d t+\boldsymbol{G}_{k}\left(\boldsymbol{X}_{k}, t\right) d \boldsymbol{W}(t)+\int_{\|\boldsymbol{X}\|<\varepsilon} \boldsymbol{\Phi}\left(\boldsymbol{X}_{k}\left(t^{-}\right), t, \boldsymbol{\xi}\right) \widetilde{\boldsymbol{N}}(d t, d \boldsymbol{\xi})
$$

for any $\boldsymbol{X}_{k}\left(t_{0}\right)=\boldsymbol{X}_{0} \in \mathbb{R}^{n}$. Define a stopping time

$$
s_{k}=\inf \left\{t \geq t_{0}:\left\|\boldsymbol{X}_{k}(t)\right\|>k\right\}
$$

where we let $\inf \emptyset=\infty$ as usual. Obviously for any $\sigma<s_{k}$, we have $\left\|\boldsymbol{X}_{k}(\sigma)\right\| \leq k$. From definition of $\boldsymbol{F}_{k}(\boldsymbol{X}, t), \boldsymbol{G}_{k}(\boldsymbol{X}, t)$, and $\boldsymbol{\Phi}_{k}\left(\boldsymbol{X}, t^{-}, \boldsymbol{\xi}\right)$ in (A1), it is seen that

$$
\begin{aligned}
& \boldsymbol{F}_{k+1}\left(\boldsymbol{X}_{k}(\sigma), t\right)=\boldsymbol{F}_{k}\left(\boldsymbol{X}_{k}(\sigma), t\right), \\
& \boldsymbol{G}_{k+1}\left(\boldsymbol{X}_{k}(\sigma), t\right)=\boldsymbol{G}_{k}\left(\boldsymbol{X}_{k}(\sigma), t\right), \\
& \boldsymbol{\Phi}_{k+1}\left(\boldsymbol{X}_{k}(\sigma), t, \boldsymbol{\xi}\right)=\boldsymbol{\Phi}_{k}\left(\boldsymbol{X}_{k}(\sigma), t, \boldsymbol{\xi}\right),
\end{aligned}
$$

for all $t_{0} \leq \sigma<s_{k}$. From (A2) and (A4), it holds that

$$
\boldsymbol{X}_{k+1}(t)=\boldsymbol{X}_{k}(t), \quad \forall t_{0} \leq t \leq s_{k} .
$$

This further implies that $s_{k}$ is increasing in $k$. Thus, we can define $s=\lim _{k \rightarrow \infty} s_{k}$. From (A5), we also have

$$
\boldsymbol{X}(t)=\boldsymbol{X}_{k}(t), \quad \forall t_{0} \leq t<s_{k} .
$$

This together with (A4) means that $\boldsymbol{X}(t)$ is the unique solution of (1) for $t \in\left[t_{0}, s_{k}\right)$. We need to show that $\mathbb{P}(s=\infty)=1$. Applying the Itô formula to $U\left(\boldsymbol{X}_{k}, t\right)$ with $\boldsymbol{X}_{k}$ the solution of (A2) gives $\begin{aligned} U\left(\boldsymbol{X}_{k}\left(t \wedge s_{k}\right), t\right) & =U\left(\boldsymbol{X}_{0}, t_{0}\right)+\int_{t_{0}}^{t \wedge s_{k}} \mathcal{L}_{k} U\left(\boldsymbol{X}_{k}(\sigma), \sigma\right) d \sigma+\int_{t_{0}}^{t \wedge s_{k}} U_{\boldsymbol{X}_{k}}\left(\boldsymbol{X}_{k}(\sigma), \sigma\right) \boldsymbol{G}_{k}\left(\boldsymbol{X}_{k}(\sigma), \sigma\right) d \boldsymbol{W}(\sigma) \\ & +\int_{t_{0}}^{t \wedge s_{k}} \sum_{\ell=1}^{m_{2}} \int_{\left|\xi_{\ell}\right|<\varepsilon_{\ell}}\left[U\left(\boldsymbol{X}_{k}\left(\sigma^{-}\right)+\boldsymbol{\Phi}^{(\ell)}\left(\boldsymbol{X}_{k}\left(\sigma^{-}\right), \sigma, \xi_{\ell}\right), \sigma\right)-U\left(\boldsymbol{X}_{k}\left(\sigma^{-}\right), \sigma\right)\right] \widetilde{N}_{\ell}\left(d \sigma, d \xi_{\ell}\right),\end{aligned}$

where

$$
\begin{aligned}
& \mathcal{L}_{k} U\left(\boldsymbol{X}_{k}, \sigma\right)=U_{\sigma}\left(\boldsymbol{X}_{k}, \sigma\right)+\boldsymbol{F}_{k}\left(\boldsymbol{X}_{k}, \sigma\right) U_{\boldsymbol{X}_{k}}\left(\boldsymbol{X}_{k}, \sigma\right)+\frac{1}{2} \operatorname{Tr}\left(\boldsymbol{G}_{k}^{T}\left(\boldsymbol{X}_{k}, \sigma\right) U_{\boldsymbol{X}_{k} \boldsymbol{X}_{k}}(\boldsymbol{X}, \sigma) \boldsymbol{G}_{k}\left(\boldsymbol{X}_{k}, \sigma\right)\right) \\
& +\sum_{\ell=1}^{m_{2}} \int_{\left|\xi_{\ell}\right|<\varepsilon_{\ell}}\left[U\left(\boldsymbol{X}_{k}\left(\sigma^{-}\right)+\boldsymbol{\Phi}^{(\ell)}\left(\boldsymbol{X}_{k}\left(\sigma^{-}\right), \sigma, \xi_{\ell}\right), \sigma\right)-U\left(\boldsymbol{X}_{k}\left(\sigma^{-}\right), \sigma\right)\right. \\
& \left.-U_{\boldsymbol{X}_{k}}\left(\boldsymbol{X}_{k}\left(\sigma^{-}\right), \sigma\right) \boldsymbol{\Phi}^{(\ell)}\left(\boldsymbol{X}_{k}\left(\sigma^{-}\right), \sigma, \xi_{\ell}\right)\right] \nu_{\ell}\left(d \xi_{\ell}\right) \text {. }
\end{aligned}
$$

By definition of $\boldsymbol{F}_{k}, \boldsymbol{G}_{k}$, and $\boldsymbol{\Phi}_{k}$ in (A1), we have

$$
\mathcal{L}_{k} U\left(\boldsymbol{X}_{k}(\sigma), \sigma\right)=\mathcal{L} U\left(\boldsymbol{X}_{k}(\sigma), \sigma\right), \forall \sigma<t \wedge s_{k},
$$

where $\mathcal{L} U\left(\boldsymbol{X}_{k}(\sigma), \sigma\right)=\left.\mathcal{L} U(\boldsymbol{X}(\sigma), \sigma)\right|_{\boldsymbol{X}=\boldsymbol{X}_{k}}$. Moreover, we have

$$
\mathbb{E}\left\{\int_{t_{0}}^{t \wedge s_{k}} U_{\boldsymbol{X}_{k}}\left(\boldsymbol{X}_{k}(\sigma), \sigma\right) \boldsymbol{G}_{k}\left(\boldsymbol{X}_{k}(\sigma), \sigma\right) d \boldsymbol{W}(\sigma)\right\}=0,
$$

which implies that

$$
\mathbb{E}\left\{\int_{t_{0}}^{t \wedge s_{k}} U_{\boldsymbol{X}_{k}}\left(\boldsymbol{X}_{k}(\sigma), \sigma\right) \boldsymbol{G}\left(\boldsymbol{X}_{k}(\sigma), \sigma\right) d \boldsymbol{W}(\sigma)\right\}=0
$$


Thus, taking expectancy of (A7) and using (7) gives

$$
\begin{aligned}
\mathbb{E}\left\{U\left(\boldsymbol{X}_{k}\left(t \wedge s_{k}\right), t\right)\right\} & \leq \mathbb{E}\left\{U\left(\boldsymbol{X}_{0}, t_{0}\right)\right\}+c_{3} \mathbb{E}\left\{\int_{t_{0}}^{t \wedge s_{k}}\left(1+U\left(\boldsymbol{X}_{k}(\sigma), \sigma\right)\right) d \sigma\right\} \\
& \leq \mathbb{E}\left\{U\left(\boldsymbol{X}_{0}, t_{0}\right)\right\}+c_{3} \mathbb{E}\left\{\int_{t_{0}}^{t}\left(1+U\left(\boldsymbol{X}_{k}\left(\sigma \wedge s_{k}\right), \sigma\right)\right) d \sigma\right\} .
\end{aligned}
$$

Applying the Gronwall inequality to (A12) yields

$$
\mathbb{E}\left\{U\left(\boldsymbol{X}_{k}\left(t \wedge s_{k}\right), t\right)\right\} \leq\left(c_{3}\left(t-t_{0}\right)+\mathbb{E}\left\{U\left(\boldsymbol{X}_{0}, t_{0}\right)\right\}\right) e^{c_{3}\left(t-t_{0}\right)},
$$

which further gives

$$
\begin{aligned}
\inf _{\|\boldsymbol{X}\| \geq k} U(\boldsymbol{X}, t) \mathbb{P}\left(s_{k} \leq t\right) & \leq \mathbb{E}\left\{U\left(\boldsymbol{X}_{k}\left(s_{k}\right), t\right) \boldsymbol{I}_{\left.s_{k} \leq t\right\}}\right\} \\
& \leq\left(c_{3}\left(t-t_{0}\right)+\mathbb{E}\left\{U\left(\boldsymbol{X}_{0}, t_{0}\right)\right\}\right) e^{c_{3}\left(t-t_{0}\right)}
\end{aligned}
$$

Therefore

$$
\mathbb{P}\left(s_{k} \leq t\right) \leq \frac{\left(c_{3}\left(t-t_{0}\right)+\mathbb{E}\left\{U\left(\boldsymbol{X}_{0}, t_{0}\right)\right\}\right) e^{c_{3}\left(t-t_{0}\right)}}{\inf _{\|\boldsymbol{X}\| \geq k} U(\boldsymbol{X}, t)} .
$$

Letting $k$ tend to infinity and using (6) result in $\mathbb{P}\left(s_{k} \leq t\right)=0$. Since $t$ is arbitrary, $\mathbb{P}\left(s_{k}=\infty\right)=$ $1 \Rightarrow \mathbb{P}(s=\infty)=1$ by definition $s=\lim _{k \rightarrow \infty} s_{k}$.

\section{A.2 Proof of Item 2) of Theorem 2.1}

Since all the conditions of Item 1) satisfy, there exists a global unique solution to (1). The technique in (H. Deng et al., 2001) is used at places in our proof. Applying the Itô formula to $U(\boldsymbol{X}, t)$ and the solution $\boldsymbol{X}(t)$ of (1) and using Condition (8) gives

$$
\begin{aligned}
U(\boldsymbol{X}(t), t)= & U(\boldsymbol{X}(s), s)+\int_{s}^{t} \mathscr{L} U(\boldsymbol{X}(r), r) d r+\int_{s}^{t} U_{\boldsymbol{X}}(\boldsymbol{X}(r), r) \boldsymbol{G}(\boldsymbol{X}(r), r) d \boldsymbol{W}(r) \\
& +\int_{s}^{t} \sum_{\ell=1}^{m_{2}} \int_{\left|\xi_{\ell}\right|<\varepsilon_{\ell}}\left[U\left(\boldsymbol{X}\left(r^{-}\right)+\boldsymbol{\Phi}^{(\ell)}\left(\boldsymbol{X}\left(r^{-}\right), r, \xi_{\ell}\right), r\right)-U\left(\boldsymbol{X}\left(r^{-}\right), r\right)\right] \widetilde{N}_{\ell}\left(d r, d \xi_{\ell}\right) \\
\leq & U(\boldsymbol{X}(s), s)+\int_{s}^{t}\left(-c_{4} U(\boldsymbol{X}(r), r)+c_{0}\right) d r+\int_{s}^{t} U_{\boldsymbol{X}}(\boldsymbol{X}(r), r) \boldsymbol{G}(\boldsymbol{X}(r), r) d \boldsymbol{W}(r) \\
& +\int_{s}^{t} \sum_{\ell=1}^{m_{2}} \int_{\left|\xi_{\ell}\right|<\varepsilon_{\ell}}\left[U\left(\boldsymbol{X}\left(r^{-}\right)+\boldsymbol{\Phi}^{(\ell)}\left(\boldsymbol{X}\left(r^{-}\right), r, \boldsymbol{\xi}\right), r\right)-U\left(\boldsymbol{X}\left(r^{-}\right), r\right)\right] \widetilde{N}_{\ell}\left(d r, d \xi_{\ell}\right),
\end{aligned}
$$

To further consider (A16), let us define an increasing time sequence $t_{k}, k=1, \cdots, \infty$ such that $t_{\infty}=\infty$. In each time interval $\left[t_{k-1}, t_{k}\right]$, we show that the system (1) is stable in probability for any initial value $\boldsymbol{X}_{t_{k-1}}$. For $r \in\left[t_{k-1}, t_{k}\right]$, there are two cases:

Case I: $\int_{t_{k-1}}^{t_{k}}\left(-c_{4} U(\boldsymbol{X}(r), r)+c_{0}\right) d r>0$

Case II: $\quad \int_{t_{k-1}}^{t_{k}}\left(-c_{4} U(\boldsymbol{X}(r), r)+c_{0}\right) d r \leq 0$,

Case I already implies that the system (1) is stable in probability in the interval $\left[t_{k-1}, t_{k}\right]$ due to (6). We now address Case II. For this case, we can write (A16) as

$$
\begin{aligned}
& U\left(\boldsymbol{X}\left(t_{k}\right), t_{k}\right) \leq U\left(\boldsymbol{X}\left(t_{k-1}\right), t_{k-1}\right)+\int_{t_{k-1}}^{t_{k}} U_{\boldsymbol{X}}(\boldsymbol{X}(r), r) \boldsymbol{G}(\boldsymbol{X}(r), r) d \boldsymbol{W}(r) \\
& \quad+\int_{t_{k-1}}^{t_{k}} \sum_{\ell=1}^{m_{2}} \int_{\left|\xi_{\ell}\right|<\varepsilon_{\ell}}\left[U\left(\boldsymbol{X}\left(r^{-}\right)+\boldsymbol{\Phi}^{(\ell)}\left(\boldsymbol{X}\left(r^{-}\right), r, \boldsymbol{\xi}\right), r\right)-U\left(\boldsymbol{X}\left(r^{-}\right), r\right)\right] \widetilde{N}_{\ell}\left(d r, d \xi_{\ell}\right),
\end{aligned}
$$

which implies that $U\left(\boldsymbol{X}\left(t_{k}\right), t_{k}\right)$ is a supermartingale with respect to the filtration generated by $\boldsymbol{W}(\cdot)$ and $\tilde{N}_{\ell}(\cdot, \cdot), \ell=1, \ldots, m_{2}$. By the supermartingale inequality (X. Mao, 2007, Theorem 3.6), for any class $\mathcal{K}_{\infty}$-function $\delta(\cdot)$, we have

$$
\mathbb{P}\left\{\sup _{t_{k-1} \leq s \leq t_{k}} U(\boldsymbol{X}(s), s) \geq \delta\left(U\left(\boldsymbol{X}_{k-1}, t_{k-1}\right)\right)\right\} \leq \frac{U\left(\boldsymbol{X}_{k-1}, t_{k-1}\right)}{\delta\left(U\left(\boldsymbol{X}_{k-1}, t_{k-1}\right)\right)},
$$


where $\boldsymbol{X}_{k-1}:=\boldsymbol{X}\left(t_{k-1}\right)$. Thus,

$$
\mathbb{P}\left\{\sup _{t_{k-1} \leq s \leq t_{k}} U(\boldsymbol{X}(s), s)<\delta\left(U\left(\boldsymbol{X}_{k-1}, t_{k-1}\right)\right)\right\} \geq 1-\frac{U\left(\boldsymbol{X}_{k-1}, t_{k-1}\right)}{\delta\left(U\left(\boldsymbol{X}_{k-1}, t_{k-1}\right)\right)} .
$$

Using (6) shows that $\sup _{t_{k-1} \leq s \leq t_{k}} U(\boldsymbol{X}(s), s)<\delta\left(U\left(\boldsymbol{X}_{k-1}, t_{k-1}\right)\right)$ implies that $\sup _{t_{k-1} \leq s \leq t_{k}}\|\boldsymbol{X}(s)\|<\varrho\left(\left\|\boldsymbol{X}_{k-1}\right\|\right)$, where $\varrho=\alpha_{1}^{-1} \circ \delta \circ \alpha_{2}$. For a given $\varepsilon>0$, we choose a class $\mathcal{K}_{\infty}$-function $\delta(\cdot)$ such that $\frac{U\left(\boldsymbol{X}_{k-1}, t_{k-1}\right)}{\delta\left(U\left(\boldsymbol{X}_{k-1}, t_{k-1}\right)\right)} \leq \varepsilon$. Thus, we can write (A20) as

$$
\mathbb{P}\left\{\sup _{t_{k-1} \leq s \leq t_{k}}\|\boldsymbol{X}(s)\|<\varrho\left(\left\|\boldsymbol{X}_{k-1}\right\|\right)\right\} \geq 1-\varepsilon .
$$

This further implies that

$$
\mathbb{P}\left\{\left\|\boldsymbol{X}\left(t_{k}\right)\right\|<\varrho\left(\left\|\boldsymbol{X}_{k-1}\right\|\right)\right\} \geq 1-\varepsilon .
$$

For $k=1,\left\|\boldsymbol{X}_{0}\right\|$ is a.s. bounded. Combining Case I defined in (A17) and (A22) yields practical stability of (1) in probability in the interval $\left[t_{0}, t_{1}\right.$. Thus, $\boldsymbol{X}_{1}$ is a.s. bounded. The above procedure is repeated for each $k=2, \cdots$ to yield global practical stability of (1) in probability.

\section{A.3 Proof of Item 3) of Theorem 2.1}

Applying the Itô formula to $e^{c_{4}\left(t-t_{0}\right)} U(\boldsymbol{X}, t)$ and the solution $\boldsymbol{X}(t)$ of (1), and using (8) gives

$$
\begin{aligned}
e^{c_{4}\left(t-t_{0}\right)} U(\boldsymbol{X}(t), t) & =U\left(\boldsymbol{X}\left(t_{0}\right), t_{0}\right)+\int_{t_{0}}^{t} e^{c_{4}\left(r-t_{0}\right)}\left(\mathcal{L} U(\boldsymbol{X}(r), r)+c_{4} U(\boldsymbol{X}(r), r)\right) d r \\
& +\int_{t_{0}}^{t} e^{c_{4}\left(r-t_{0}\right)}\left[U_{\boldsymbol{X}}(\boldsymbol{X}(r), r) \boldsymbol{G}(\boldsymbol{X}(r), r) d \boldsymbol{W}(r)\right. \\
& \left.+\sum_{\ell=1}^{m_{2}} \int_{\left|\xi_{\ell}\right|<\varepsilon_{\ell}}\left(U\left(\boldsymbol{X}\left(r^{-}\right)+\boldsymbol{\Phi}^{(\ell)}\left(\boldsymbol{X}\left(r^{-}\right), r, \xi_{\ell}\right), r\right)-U\left(\boldsymbol{X}\left(r^{-}\right), r\right)\right) \widetilde{N}_{\ell}\left(d r, d \xi_{\ell}\right)\right] \\
\leq & U\left(\boldsymbol{X}\left(t_{0}\right), t_{0}\right)+c_{0} \int_{t_{0}}^{t} e^{c_{4}\left(r-t_{0}\right)} d r+\int_{t_{0}}^{t} e^{c_{4}\left(r-t_{0}\right)}\left[U_{\boldsymbol{X}}(\boldsymbol{X}(r), r) \boldsymbol{G}(\boldsymbol{X}(r), r) d \boldsymbol{W}(r)\right. \\
& \left.+\sum_{\ell=1}^{m_{2}} \int_{\left|\xi_{\ell}\right|<\varepsilon_{\ell}}\left(U\left(\boldsymbol{X}\left(r^{-}\right)+\boldsymbol{\Phi}^{(\ell)}\left(\boldsymbol{X}\left(r^{-}\right), r, \xi_{\ell}\right), r\right)-U\left(\boldsymbol{X}\left(r^{-}\right), r\right)\right) \tilde{N}_{\ell}\left(d r, d \xi_{\ell}\right)\right] .
\end{aligned}
$$

Multiplying both sides of (A23) with $e^{-c_{4}\left(t-t_{0}\right)}$ gives

$$
\begin{aligned}
U(\boldsymbol{X}(t), t) & \leq \underset{U}{U}\left(\boldsymbol{X}\left(t_{0}\right), t_{0}\right) e^{-c_{4}\left(t-t_{0}\right)}+\frac{c_{0}}{c_{4}}+\int_{t_{0}}^{t} e^{-c_{4}(t-r)}\left[U_{\boldsymbol{X}}(\boldsymbol{X}(r), r) \boldsymbol{G}(\boldsymbol{X}(r), r) d \boldsymbol{W}(r)\right. \\
& \left.+\sum_{\ell=1}^{m_{2}} \int_{\left|\xi_{\ell}\right|<\varepsilon_{\ell}}\left(U\left(\boldsymbol{X}\left(r^{-}\right)+\boldsymbol{\Phi}^{(\ell)}\left(\boldsymbol{X}\left(r^{-}\right), r, \xi_{\ell}\right), r\right)-U\left(\boldsymbol{X}\left(r^{-}\right), r\right)\right) \widetilde{N}_{\ell}\left(d r, d \xi_{\ell}\right)\right],
\end{aligned}
$$

which can be written as

$$
U(\boldsymbol{X}(t), t) \leq U\left(\boldsymbol{X}\left(t_{0}\right), t_{0}\right) e^{-c_{4}\left(t-t_{0}\right)}+\frac{c_{0}}{c_{4}}+\sum_{\ell=1}^{m} M_{\ell}(t)
$$

where

$$
M_{\ell}(t):=\int_{t_{0}}^{t} \bar{g}_{\ell}(\boldsymbol{X}(r), r) d W_{\ell}(r)+\int_{t_{0}}^{t} \int_{\left|\xi_{\ell}\right|<\varepsilon_{\ell}} \bar{\phi}_{\ell}\left(\boldsymbol{X}\left(r^{-}\right), r, \xi_{\ell}\right) \widetilde{N}_{\ell}\left(d r, d \xi_{\ell}\right),
$$

and

$$
\begin{aligned}
& m=m_{1} \vee m_{2}, \\
& U_{\boldsymbol{X}}(\boldsymbol{X}(r), r) \boldsymbol{G}(\boldsymbol{X}(r), r)=e^{-c_{4}(t-r)} \operatorname{row}\left(\bar{g}_{1}(\boldsymbol{X}(r), r)\right), \cdots, \bar{g}_{\ell}(\boldsymbol{X}(r), r), \cdots, \bar{g}_{m}(\boldsymbol{X}(r), r), \\
& \bar{\phi}_{\ell}\left(\boldsymbol{X}(r), r, \xi_{\ell}\right)=e^{-c_{4}(t-r)}\left(U\left(\boldsymbol{X}(r)+\boldsymbol{\Phi}^{(\ell)}\left(\boldsymbol{X}(r), r, \xi_{\ell}\right), r\right)-U(\boldsymbol{X}(r), r)\right),
\end{aligned}
$$

and if $m_{1}<m$, we set $\bar{g}_{\ell}=0$ and $W_{\ell}=W_{m_{1}}$ for $\ell=m_{1}, \cdots, m$; if $m_{2}<m$, we set $\bar{\phi}_{\ell}=0$ and $\widetilde{N}_{\ell}=\widetilde{N}_{m_{2}}$ for $\ell=m_{2}, \cdots, m$. Since we have already proved that the system (1) is globally practically stable in probability, $\boldsymbol{G}(\boldsymbol{X}, t)$ and $\boldsymbol{\Phi}(\boldsymbol{X}, t, \boldsymbol{\xi})$ satisfy Conditions $\mathrm{C} 1$ and $\mathrm{C} 2$ of Assumption 2.1, $U(\boldsymbol{X}, t) \leq \alpha_{2}\left(\|\boldsymbol{X}\|^{p}\right)$, see Condition (6), and $\alpha_{2}\left(\left\|\boldsymbol{X}+\boldsymbol{\Phi}^{\ell}\right\|^{p}\right) \leq$ 
$\alpha_{2}\left(2^{p-1}\left(\|\boldsymbol{X}\|^{p}+\left\|\boldsymbol{\Phi}^{\ell}\right\|^{p}\right)\right) \leq \bar{\alpha}_{21}\left(\|\boldsymbol{X}\|^{p}\right)+\bar{\alpha}_{22}\left(\left\|\boldsymbol{\Phi}^{\ell}\right\|^{p}\right)$ with $\bar{\alpha}_{21}$ and $\bar{\alpha}_{22}$ being some class $\mathcal{K}_{\infty^{-}}$ functions, we have $\int_{t_{0}}^{t}\left|\bar{g}_{\ell}(\boldsymbol{X}(r), r)\right|^{2} d r<\infty$ and $\int_{t_{0}}^{t} \int_{\left|\xi_{\ell}\right|<\varepsilon_{\ell}}\left|\bar{\phi}_{\ell}\left(\boldsymbol{X}\left(r^{-}\right), r, \xi_{\ell}\right)\right|^{2} \nu_{\ell}\left(d \xi_{\ell}\right) d r<\infty$ a.s. for all $t \in\left[t_{0}, \infty\right)$. Thus, we can apply the exponential martingale inequality, see (Applebaum, 2009, Theorem 5.2.9), to $M_{\ell}(t)$ with $T=k, a=\epsilon, b=\epsilon k$, where $\epsilon \in\left(0, \frac{1}{2}\right)$ and $k \geq t_{0}$ is an integer, as follows:

$$
\begin{aligned}
\mathbb{P}\left\{\operatorname { s u p } _ { t _ { 0 } \leq t \leq k } \left[M_{\ell}(t)-\frac{\epsilon}{2} \int_{t_{0}}^{t}\left|\bar{g}_{\ell}(s)\right|^{2} d s\right.\right. & \\
& \left.\left.-\frac{1}{\epsilon} \int_{t_{0}}^{t} \int_{\left|\xi_{\ell}\right|<\varepsilon_{\ell}}\left(e^{\epsilon \bar{\phi}_{\ell}\left(\boldsymbol{X}\left(s^{-}\right), s, \xi_{\ell}\right)}-1-\epsilon \bar{\phi}_{\ell}\left(\boldsymbol{X}\left(s^{-}\right), s, \xi_{\ell}\right)\right) \nu_{\ell}\left(d \xi_{\ell}\right) d s\right]>\epsilon k\right\}<e^{-\epsilon^{2} k .} .
\end{aligned}
$$

Since $\sum_{k=1}^{\infty} e^{-\epsilon^{2} k}<\infty$, applying the Borel-Cantelli lemma to (A28) gives

$$
\begin{gathered}
\mathbb{P}\left\{\operatorname { l i m } _ { k \rightarrow \infty } \operatorname { i n f } \left(\operatorname { s u p } _ { t _ { 0 } \leq t \leq k } \left[M_{\ell}(t)-\frac{\epsilon}{2} \int_{t_{0}}^{t}\left|\bar{g}_{\ell}(s)\right|^{2} d s-\frac{1}{\epsilon} \int_{t_{0}}^{t} \int_{\left|\xi_{\ell}\right|<\varepsilon_{\ell}}\left(e^{\epsilon \bar{\phi}_{\ell}\left(\boldsymbol{X}\left(s^{-}\right), s, \xi_{\ell}\right)}-1\right.\right.\right.\right. \\
\left.\left.\left.\left.-\epsilon \bar{\phi}_{\ell}\left(\boldsymbol{X}\left(s^{-}\right), s, \xi_{\ell}\right)\right) \nu_{\ell}\left(d \xi_{\ell}\right) d s\right] \leq \epsilon k\right)\right\}=1 .
\end{gathered}
$$

Therefore, for almost all $\omega \in \Omega$ there exists a random integer $k_{0}=k_{0}(\omega)$ such that for $k \geq k_{0}$ and $t_{0} \leq t \leq n$ :

$$
M_{\ell}(t) \leq \frac{\epsilon}{2} \int_{t_{0}}^{t}\left|\bar{g}_{\ell}(s)\right|^{2} d s+\epsilon k+\frac{1}{\epsilon} \int_{t_{0}}^{t} \int_{\left|\xi_{\ell}\right|<\varepsilon_{\ell}}\left(e^{\epsilon \bar{\phi}_{\ell}\left(\boldsymbol{X}\left(s^{-}\right), s, \xi_{\ell}\right)}-1-\epsilon \bar{\phi}_{\ell}\left(\boldsymbol{X}\left(s^{-}\right), s, \xi_{\ell}\right)\right) \nu_{\ell}\left(d \xi_{\ell}\right) d s .
$$

To further consider (A30), we need the following inequality by using Taylor series expansion of $e^{x}$ for all $x \in \mathbb{R}^{+}$:

$$
\begin{aligned}
e^{x} & =1+x+\frac{x^{2}}{2 !}\left[1+\frac{2 !}{3 !} x+\frac{2 ! 2 !}{4 !} \frac{x^{2}}{2 !}+\frac{2 ! 3 !}{5 !} \frac{x^{3}}{3 !}+\cdots\right] \\
& \leq 1+x+\frac{x^{2}}{2 !} e^{x}
\end{aligned}
$$

Applying (A31) to (A30) yields

$$
M_{\ell}(t) \leq \frac{\epsilon}{2} \int_{t_{0}}^{t}\left|\bar{g}_{\ell}(s)\right|^{2} d s+\epsilon k+\epsilon \int_{t_{0}}^{t} \int_{\left|\xi_{\ell}\right|<\varepsilon_{\ell}} \bar{\phi}_{\ell}^{2}\left(\boldsymbol{X}\left(s^{-}\right), s, \xi_{\ell}\right) e^{\epsilon \bar{\phi}_{\ell}\left(\boldsymbol{X}\left(s^{-}\right), s, \xi_{\ell}\right)} \nu_{\ell}\left(d \xi_{\ell}\right) d s .
$$

Since we have proved that the system (1) is globally stable in probability, under Conditions (9), $\mathrm{C} 1$ and $\mathrm{C} 2$ of Assumption 2.1, by letting $\epsilon \rightarrow 0$ the dominated convergence theorem holds that for all $t \geq t_{0}$ :

$$
\lim _{\epsilon \rightarrow 0}\left[\frac{\epsilon}{2} \int_{t_{0}}^{t}\left|\bar{g}_{\ell}(s)\right|^{2} d s+\epsilon k+\epsilon \int_{t_{0}}^{t} \int_{\left|\xi_{\ell}\right|<\varepsilon_{\ell}} \bar{\phi}_{\ell}^{2}\left(\boldsymbol{X}\left(s^{-}\right), s, \xi_{\ell}\right) e^{\epsilon \bar{\phi}_{\ell}\left(\boldsymbol{X}\left(s^{-}\right), s, \xi_{\ell}\right)} \nu_{\ell}\left(d \xi_{\ell}\right) d s\right]=0 .
$$

Substituting (A33) into (A32) then into (A25) and using (6) gives (10).

\section{References}

Antunes, D. J., Hespanha, J. P., \& Silvestre, C. J. (2010). Impulsive systems triggered by superposed renewal processes. Proceedings of the 49th IEEE conference on decision and control, 1779-1784.

Antunes, D. J., Hespanha, J. P., \& Silvestre, C. J. (2012). Volterra integral approach to impulsive renewal systems: application to networked control. IEEE Transactions on Automatic Control, 57(3), 607-619.

Antunes, D. J., Hespanha, J. P., \& Silvestre, C. J. (2013a). Stability of networked control systems with asynchronous renewal links: An impulsive systems approach. Automatica, 49(2), 402-413.

Antunes, D. J., Hespanha, J. P., \& Silvestre, C. J. (2013b). Stochastic hybrid systems with renewal transitions: moment analysis with application to networked control systems with delays. SIAM Journal on Control and Optimization, 51(2), 1481-1499.

Antunes, D. J., Hespanha, J. P., \& Silvestre, C. J. (2014). Stochastic networked control systems with dynamic protocols. Asian Journal of Control, 16(6), 1-12.

Applebaum, D. (2009). Lévy processes and stochastic calculus (2nd ed.). Cambridge University Press.

Applebaum, D., \& Siakalli, M. (2009). Asymptotic stability of stochastic differential equations driven by Lévy noise. Journal of Applied Probability, 46, 1116-1129.

Charara, A., Miras, J. D., \& Caron, B. (1996). Nonlinear control of a magnetic levitation system without premagetization. IEEE Transactions on Control Systems Technology, 4, 513-523. 
Deng, F., Luo, Q., \& Mao, X. (2012). Stochastic stabilization of hybrid differential equations. Automatica, 48(9), 2321-2328.

Deng, H., \& Krstic, M. (1997). Stochastic nonlinear stabilization-Part I: A backstepping design. Systems and Control Letters, 32(3), 143-150.

Deng, H., Krstic, M., \& Williams, R. (2001). Stabilization of stochastic nonlinear systems driven by noise of unknown covariance. IEEE Transactions on Automatic Control, 46(8), 1237-1253.

de Queiroz, M., \& Dawson, D. (1996). Nonlinear control of active magnetic bearings: A backstepping approach. IEEE Transactions on Control Systems Technology, 14(5), 545-552.

de Queiroz, M., Dawson, D., \& Suri, A. (1998). Nonlinear control of a large-gap 2-dof magnetic bearing system based on a coupled force model. IEE Proceedings of Control Theory Applications, 145(3), 269-276.

Do, K. D. (2010). Control of nonlinear systems with output tracking error constraints and its application to magnetic bearings. International Journal of Control, 83(6), 1199-1216.

Do, K. D. (2015). Global inverse optimal stabilization of stochastic nonholonomic systems. Systems \& Control Letters, 41-55.

Do, K. D. (2016). Stability of nonlinear stochastic distributed parameter systems and its applications. Journal of Dynamic Systems, Measurement, and Control, 138, 101010-1:101010-12.

Fujita, M., Hatake, K., \& Matsumura, F. (1993). Loop shaping based robust control of a magnetic bearing. IEEE Control Systems Magazine, 13(4), 47-85.

Hespanha, J. P., \& Teel, A. R. (2006). Stochastic impulsive systems driven by renewal processes. 17th international symposium on mathematical theory of networks and systems.

Jagtap, P., \& Zamani, M. (2017). Backstepping design for incremental stability of stochastic Hamiltonian systems with jumps. IEEE Transactions on Automatic Control, In Press.

Khalil, H. (2002). Nonlinear systems. Prentice Hall.

Khasminskii, R. (1980). Stochastic stability of differential equations. Rockville MD: S\&N International.

Krstic, M., Kanellakopoulos, I., \& Kokotovic, P. (1995). Nonlinear and adaptive control design. New York: Wiley.

Liu, K. (2006). Stability of infinite dimensional stochastic differential equations with applications. Boca Raton, FL: Chapman and Hall/CRC.

Mao, W., You, S., \& Mao, X. (2016). On the asymptotic stability and numerical analysis of solutions to nonlinear stochastic differential equations with jumps. Journal of Computational and Applied Mathematics, 301, 1-15.

Mao, X. (1999). Stability of stochastic differential equations with markovian switching. Stochastic Processes and their Applications, $79(1), 45-67$.

Mao, X. (2007). Stochastic differential equations and applications (2nd ed.). Cambridge: Woodhead publishing.

Mao, X., Yin, G. G., \& Yuan, C. (2007). Stabilization and destabilization of hybrid systems of stochastic differential equations. Automatica, 43(2), 264-273.

Matsumura, F., \& Yoshimoto, T. (1986). System modeling and control design of a horizontal-shaft magneticbearing system. IEEE Transactions on Magnetics, 22(3), 196-203.

Mittal, S., \& Menq, C. (1997). Precision motion control of a magnetic sspension actuator using a robust nonlinear compensation scheme. IEEE/ASME Transactions on Mechatronics, 2, 268-280.

Mohamed, A. M., \& Emad, F. P. (1992). Conical magnetic bearings with radial and thrust control. IEEE Transactions on Automatic Control, 37(3), 1859-1869.

Nane, E., \& Ni, Y. (2017). Stability of the solution of stochastic differential equation driven by time-changed Lévy noise. Proceedings of the American Mathematical Society, 145, 3085-3104.

Smith, R. D., \& Weldon, W. F. (1995). Nonlinear control of a rigid rotor magnetic bearing system: Modeling and simulation with full state feedback. IEEE Transactions on Magnetics, 31(2), 973-980.

Stachurski, W., Midera, S., \& Kruszýnski, B. (2012). Determination of mathematical formulae for the cutting force $f_{c}$ during the turning of c45 steel. Mechanics and Mechanical Engineering, 16 (2), 73-79.

Teel, A. R., Subbaramana, A., \& Sferlazza, A. (2014). Stability analysis for stochastic hybrid systems: A survey. Automatica, 50, 2435-2456.

Torries, M., Sira-Ramirez, H., \& Escobar, G. (1999). Sliding mode nonlinear control of magnetic bearings. Proceedings of the 1999 IEEE International Conference on Control Applications, 743-748.

Woodson, H. H., \& Melcher, J. R. (1968). Electromechanical dynamics-part $i$ : Discrete systems. New York: 
Englewood Cliffs, NJ: Prentice-Hall.

Zhu, Q. (2014). Asymptotic stability in the pth moment for stochastic differential equations with Lévy noise. Journal of Mathematical Analysis and Applications, 416, 126-142. 